TI 2011-093/4

Tinbergen Institute Discussion Paper

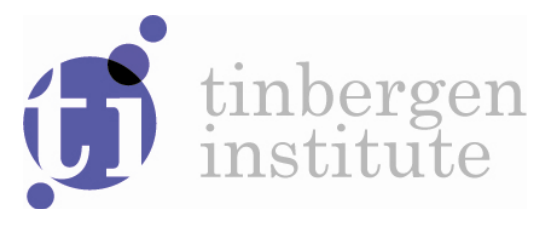

\title{
Bayesian Forecasting of Federal Funds Target Rate Decisions
}

Sjoerd van den Hauwe',2

Dick van Dijk ${ }^{1,2}$

Richard Paap ${ }^{1,2}$

' Econometric Institute, Erasmus School of Economics, Erasmus University Rotterdam;

2 Tinbergen Institute 
Tinbergen Institute is the graduate school and research institute in economics of Erasmus University Rotterdam, the University of Amsterdam and VU University Amsterdam.

More TI discussion papers can be downloaded at http://www.tinbergen.nl

Tinbergen Institute has two locations:

Tinbergen Institute Amsterdam

Gustav Mahlerplein 117

1082 MS Amsterdam

The Netherlands

Tel.: +31(0)205251600

Tinbergen Institute Rotterdam

Burg. Oudlaan 50

3062 PA Rotterdam

The Netherlands

Tel.: +31(0)10 4088900

Fax: +31(0)104089031

Duisenberg school of finance is a collaboration of the Dutch financial sector and universities, with the ambition to support innovative research and offer top quality academic education in core areas of finance.

DSF research papers can be downloaded at: http://www.dsf.nl/

Duisenberg school of finance

Gustav Mahlerplein 117

1082 MS Amsterdam

The Netherlands

Tel.: +31(0)20 5258579 


\title{
Bayesian Forecasting of Federal Funds Target Rate Decisions
}

\author{
Sjoerd van den Hauwe ${ }^{1,2} \quad$ Richard Paap ${ }^{1,2} \quad$ Dick van Dijk $k^{1,2 *}$ \\ ${ }^{1}$ Econometric Institute \\ Erasmus University Rotterdam \\ ${ }^{2}$ Tinbergen Institute \\ July 13, 2011
}

\begin{abstract}
This paper examines which macroeconomic and financial variables are most informative for the federal funds target rate decisions made by the Federal Open Market Committee (FOMC) from a forecasting perspective. The analysis is conducted for the FOMC decision during the period January 1990 - June 2008, using dynamic ordered probit models with a Bayesian endogenous variable selection methodology and real-time data for a set of 33 candidate predictor variables. We find that indicators of economic activity and forward-looking term structure variables as well as survey measures have most predictive ability. For the full sample period, in-sample probability forecasts achieve a hitrate of 90 percent. Based on out-of-sample forecasts for the period January 2001 - June 2008, 82 percent of the FOMC decisions are predicted correctly.
\end{abstract}

Keywords: Federal funds target rate, real-time forecasting, dynamic ordered probit, variable selection, Bayesian analysis, importance sampling.

JEL Classification: E52, E58, C25, C11, C53.

*Corresponding author: Dick van Dijk, Econometric Institute, Erasmus University Rotterdam, P.O. Box 1738, NL-3000 DR Rotterdam, The Netherlands. Tel.: +31-10-40891263, Fax: +31-104089162. E-mail address: djvandijk@ese.eur.nl 


\section{Introduction}

The federal funds target rate is one of the key monetary policy instruments of the Federal Reserve and, thereby, an important indicator of the (future) state of the U.S. economy. Not surprisingly then, decisions concerning the target rate as made by the Federal Open Market Committee (FOMC) are closely watched by investors, firms, and other economic agents. Likewise, speeches, interviews, and other types of communication by FOMC members are routinely scrutinized for information about future target rate decisions. Surprises in target rate decisions have been documented to have a pronounced impact on financial markets, see Bernanke and Kuttner (2005), Faust et al. (2007), Andersen et al. (2007), and Chulia-Soler et al. (2010), among many others.

Federal funds target rate decisions are made by the FOMC during their meetings held approximately every six weeks, and are the outcome of a complicated decisionmaking process. The target rate is set as a guideline for the Federal Reserve's open market operations, that is, purchases and sales of U.S. Treasury and federal agency securities, which is one of the Fed's principal tools for implementing its monetary policy (in addition to the discount window and reserve requirements). Numerous economic indicators are closely monitored by the FOMC, in order to determine the most appropriate course of action. Most attention is believed to be paid to inflation (in deviation from a target) and the output gap, in accordance with the main goals of the Federal Reserve's monetary policy to promote price stability and maximum sustainable output growth and employment (Federal Reserve Board of Governors; 2005), as formalized in the Taylor rule, see Taylor (1993). The minutes of FOMC meetings indicate, however, that a large number of other economic variables, reflecting developments in the labor market, housing market, and financial markets, also play a substantial role in the considerations. ${ }^{1}$

The aim of this paper is to assess which macroeconomic and financial variables are most informative for the FOMC's federal funds target rate decisions from a

\footnotetext{
${ }^{1}$ For example, the following variables, among others, are explicitly mentioned in the minutes of the FOMC meeting held on December 15-16, 2008: private payrolls, unemployment rate, new claims for unemployment insurance, industrial production, factory utilization rate, consumer spending, consumer sentiment, new building permits, house prices, vacancy rates, new orders for nondefense capital goods, manufacturing and wholesale trade inventories, purchasing managers' index, core consumer price inflation, energy prices, consumer food prices, inflation expectations, stock prices, bond yields and spreads.
} 
forecasting perspective. We analyze the 157 target rate decisions made during the period January 1990 - June 2008, and consider a set of 33 possible predictors. The variables in this set are classified into three categories. First, we include recent releases of macro variables such as output, employment and inflation, reflecting the fact that these are most directly related to the Federal Reserve's monetary policy goals. Second, we examine the information embedded in recent realizations of several other macroeconomic and financial variables. Most of the macro variables in this group are established leading indicators, providing signals about future economic developments that are potentially useful for predicting FOMC decisions. Similarly, the forward-looking nature of asset prices such as stock prices and interest rates has been shown to result in predictive ability for macro variables such as output and inflation, see Stock and Watson (2003), among others. It seems natural to examine whether this also holds for FOMC target rate decisions. Third, we include survey measures of consumer confidence and expectations as well as professional forecasts for inflation, output and interest rates. This is motivated by the results in Ang et al. (2007), who demonstrate that survey-based measures and forecasts outperform macro variables and asset prices in forecasting inflation.

In our analysis we employ ordered probit models, to take into account that actual target rate decisions are discrete, in the sense that, with few exceptions, they occur in multiples of 25 basis points. We limit ourselves to modeling and forecasting the sign of the target rate decisions (or, in economic terms, the direction of monetary policy), making no distinction between changes of different magnitudes.

Visual inspection of the time series of the federal funds target rate shows that decisions of a given type come in clusters. This may reflect persistence in shocks to the macroeconomic variables that drive monetary policy in general and the FOMC decisions in particular. Often, however, this feature is interpreted as a sign of interest rate smoothing by the FOMC. Several reasons for this 'inertia' in monetary policy decisions have been put forward. These include uncertainty about the true structure of the economy and uncertainty about the accuracy of initial data releases of important macro variables, in particular output and employment. A third reason is the possibility to influence market expectations if monetary policymakers demonstrate that they (are willing to) implement a consistent, long-run interest rate policy, see Dueker and Rasche (2004) for a discussion. Rudebusch (2002) suggests that much of the evidence for interest rate smoothing may in fact be the result of omitting some relevant determinants of target rate decisions from the model. As 
it is not really possible to include all relevant variables, empirically it is difficult to distinguish between policy inertia and persistence of macroeconomic shocks. While economically this distinction is important for the interpretation of the clustering of similar target rate decisions, from a forecasting perspective the true explanation is less crucial. Following Rudebusch (2002), we simply allow for autocorrelated errors to capture the temporal dependence in the FOMC decisions. In the empirical analysis we compare this dynamic ordered probit model with a static version, in order to assess the importance of explicitly accounting for the observed clustering.

FOMC target rate decisions, and possible determinants thereof, have been studied previously by means of (dynamic) ordered probit and logit models by Dueker (1999), Vanderhart (2000), Hamilton and Jordà (2002), Dueker and Rasche (2004), $\mathrm{Hu}$ and Phillips (2004), Piazzesi (2005), Grammig and Kehrle (2008), Kim et al. (2009), Hayo and Neuenkirch (2010), Monokroussos (2011) and Kauppi (forthcoming), among others. We make three contributions to the existing literature. First, previous studies consider a pre-determined set of explanatory variables. We, however, explicitly address the question which macroeconomic and financial variables bear most predictive content for target rate decisions. This is made possible by adopting a Bayesian approach for inference and forecasting. In particular, we employ an endogenous variable selection algorithm developed by Kuo and Mallick (1998), which for each candidate predictor renders a probability that it should be included in the forecasting model. Alternatively, we can interpret this procedure as a form of Bayesian model averaging. In terms of forecasting, model uncertainty is accounted for by averaging across different models, based on the posterior probabilities of inclusion of the different predictors.

Second, while most of the previous literature on target rate decisions only considers the in-sample fit of ordered probit (or other) models, we explicitly focus on out-of-sample forecasting. For this purpose, we update the parameter beliefs each time the outcome of a new FOMC meeting is observed by employing a recursive importance sampling scheme. As we integrate with respect to the updated posterior beliefs, our real-time forecasts do not only account for model uncertainty but also for parameter uncertainty.

Third, we take care to construct our probability forecasts of the target rate decisions in real time, in order to mimic the FOMC decision making as realistically as possible. In addition to the recursive updating of the parameter estimates mentioned above, this means that we account for the fact that many macroeconomic 
variables are revised after their initial release. Hence, the values of variables like output, employment and inflation as they are available to us now are not necessarily the same as available to the FOMC members in the past, at the time of their target rate decisions. Neglecting this aspect may crucially affect the results of historical forecasting exercises as conducted here, see Diebold and Rudebusch (1991), Rudebusch (2001), Stark and Croushore (2002), and Croushore (2006), among others. In order to address this issue we put together a real-time data set for the macroeconomic variables that we consider as possible predictors of the target rate decisions. This data set enables us to use the values of these variables as they actually were available to the FOMC at each point in time.

Our main empirical results are as follows. First, we find that measures of recent (changes in) economic activity like the output gap and industrial production, which are closely linked to the Federal Reserve's monetary policy goals, indeed have substantial predictive content for the FOMC target rate decisions. Perhaps surprisingly, the predictive ability of past inflation is much more limited. The most important individual predictor is the spread between the six-month T-bill rate and the effective federal funds rate, reflecting the forward-looking nature of the term structure. In agreement with Ang et al. (2007), we find that survey-based measures and forecasts contribute to forecasting the FOMC decisions. This holds in particular for consumer confidence, expectations about the labor market and term structure forecasts. Second, the dynamic ordered probit model with endogenous variable selection correctly predicts no less than 90 percent of the target rate decisions in-sample (during the complete sample period from January 1990 until June 2008) and 82 percent during the out-of-sample period January 2001 - June 2008. It is crucially important to incorporate the clustering of similar target rate decisions in the model. Compared to the hit rates of 90 and 82 percent for the in-sample and out-of-sample forecasts achieved by the dynamic model, the static model predicts 74 and 77 percent of the target rate decisions correctly. Finally, using real-time data instead of fully revised data (as available at the time of the analysis) does not lead to deterioration in forecasting performance. Using fully revised data, the dynamic probit model produces forecasts that result in hit rates of 89 and 81 percent.

The outline of the paper is as follows. In Section 2 we describe the dynamic ordered probit model with endogenous variable selection for the FOMC target rate decisions. Subsequently we discuss the Bayesian procedure for inference and realtime forecasting. In Section 3 we summarize the main features of the federal funds 
target rate during the sample period January 1990 - June 2008, and introduce the data set of candidate predictors and its real-time properties. We discuss the estimation and forecasting results in Section 4, and conclude in Section 5.

\section{Methodology}

In this section we discuss the dynamic probit model that we use to describe the discrete federal funds target rate changes. Section 2.1 deals with model specification. We opt for a Bayesian approach for estimation and inference. We consider the prior for the model parameters in Section 2.2 and discuss the main aspects of our posterior simulation scheme in Section 2.3. Finally, in Section 2.4, we outline our procedure for obtaining real-time Bayesian forecasts. Full details of the computational procedures for the posterior simulation and forecasting are provided in Appendices A and B.

\subsection{Modeling target rate changes}

As mentioned in the introduction, federal funds target rate decisions are (mostly) made during the scheduled meetings of the FOMC. These are held approximately every six weeks, in total eight times per year. Our dynamic probit modeling framework is not specified in 'meeting time' (as in Hamilton and Jordà (2002) and Hayo and Neuenkirch (2010)) though, but in calendar time with a monthly frequency, as in $\mathrm{Hu}$ and Phillips (2004) and Kim et al. (2009). A crucial difference with these studies is that we do not discard months without an FOMC meeting, as discussed in detail below.

We define $r_{t}$ as the prevailing target rate at the end of month $t$ for $t=1, \ldots, T$. As we aim to model the direction of FOMC target rate decisions we take the sign of $\Delta r_{t}=r_{t}-r_{t-1}$ as our variable of interest. We construct the discrete dependent variable $y_{t}$ according to the classification

$$
y_{t}= \begin{cases}1 & \text { if } \Delta r_{t}<0 \text { (target rate decrease) } \\ 2 & \text { if } \left.\Delta r_{t}=0 \text { (no change }\right) \\ 3 & \text { if } \Delta r_{t}>0 \text { (target rate increase) }\end{cases}
$$

This variable summarizes the FOMC decisions concerning the target rate as decided upon during its meetings and therefore is necessarily missing if there is no meeting 
in month $t .^{2}$ To describe the discrete and ordinal nature of $y_{t}$ we introduce a latent continuous variable $r_{t}^{*},(t=1, \ldots, T)$, which drives the classification, see Albert and Chib (1993) and Geweke (2005), for example. The introduction of the latent variable has two justifications. First, it facilitates the link of $y_{t}$ with potentially explanatory variables. Second, we can conveniently interpret $r_{t}^{*}$ as the publicly unobserved target rate that is central to the FOMC in choosing its policy. With our application in mind, if in month $t$ the (absolute) deviation between the previously announced target rate $r_{t-1}$ and the latent 'desired' target $r_{t}^{*}$ becomes too large, a new target is announced. Formally this decision rule, establishing the link between $r_{t}, y_{t}$ and $r_{t}^{*}$, becomes

$$
y_{t}=j \Leftrightarrow r_{t}^{*}-r_{t-1} \in\left(\alpha_{j-1}, \alpha_{j}\right], \quad \text { for } j=1,2,3,
$$

where the $\alpha_{j}$ 's are threshold parameters satisfying the restriction $-\infty=\alpha_{0}<\alpha_{1}<$ $\alpha_{2}<\alpha_{3}=\infty$. Hence, if, for example, $r_{t}^{*}$ is higher than $r_{t-1}$ by an amount that exceeds $\alpha_{2}$, the target rate is increased in month $t$ such that $r_{t}>r_{t-1}$.

With the introduction of $r_{t}^{*}$, we model the FOMC decisions by describing the behavior of this latent variable. In particular, for predicting future decisions we specify its link with macroeconomic and financial information that is available at the moment of constructing the forecast. We assume a linear relation between the unobserved target rate $r_{t}^{*}$ and covariates summarized in the vector $\mathbf{x}_{t}=\left(x_{1 t}, \ldots, x_{K t}\right)^{\prime}$, such that

$$
r_{t}^{*}=\boldsymbol{\beta}^{\prime} \mathbf{x}_{t}+u_{t}
$$

in which $\boldsymbol{\beta}=\left(\beta_{1}, \ldots, \beta_{K}\right)^{\prime}$ and $\left\{u_{t}\right\}$ is an unobserved random process. We stress that in the implementation we require that information included in $\mathbf{x}_{t}$ is available at the end of month $t-1$ (including publication lags), such that the model can indeed be used for real-time prediction of the target rate decision in month $t$. Note that our approach is conservative, in the sense that the FOMC may have had more recent information at its disposal when making the target rate decision in month $t$.

As discussed in the introduction, the FOMC monitors a large number of macroeconomic and financial variables to guide its target rate decisions, in addition to

\footnotetext{
${ }^{2}$ An alternative definition of the dependent variable $y_{t}$ disregards the meetings-issue and has an observation each month. More in particular, if there is no meeting the target rate is said to remain constant and $y_{t}$ is set equal to the value 2 for these months, as in Dueker (1999). We discuss this alternative model specification with its different interpretation and limitations, together with its estimation results in Appendix C.
} 
inflation and output measures that are directly related to monetary policy objectives. This raises the question which economic indicators are the most important predictors for the FOMC decisions. We incorporate this uncertainty with respect to the exact content of the vector $\mathbf{x}_{t}$ in our analysis of the ordered probit model. This approach has two advantages. First, it allows us to do inference on which economic variables are important determinants of the Fed's monetary policy decisions. Second, when constructing forecasts of future target rate decisions, we can account for this model uncertainty by averaging over different models with different combinations of predictor variables.

We follow the approach of Kuo and Mallick (1998) because it is computationally easy to implement and does not require extensive tuning as, for example, the method proposed by George and McCulloch (1993). We have $K$ potential predictors $x_{k t}$, $(k=1, \ldots, K)$. For describing the selection of these covariates in the model we introduce $K$ additional binary parameters $\gamma_{k},(k=1, \ldots, K)$, indicating whether the $k$-th variable is included in the model $\left(\gamma_{k}=1\right)$ or not $\left(\gamma_{k}=0\right)$. Effectively we decompose the regression parameters $\beta_{k}$ in (3) as $\beta_{k}=\psi_{k} \cdot \gamma_{k}$ with $\gamma_{k} \in\{0,1\}$ and $\psi_{k}$ denoting the effect of $x_{k t}$ on the latent target rate when it is included in the model. Modeling the uncertainty regarding relevant predictors this way boils down to determining which of the $2^{K}$ different predictor combinations are most likely to have predictive power for the target rate decisions.

The final part of our model specification concerns the distributional assumptions on $u_{t}$ in $(3) .{ }^{3}$ In order to deal with decision smoothing and policy inertia, we allow for temporal dependence in the process for $u_{t}$. In particular, we assume a Gaussian first-order autoregressive structure:

$$
u_{t}=\varphi u_{t-1}+\varepsilon_{t}, \quad \varepsilon_{t} \stackrel{i . i . d .}{\sim} \mathcal{N}(0,1) .
$$

A similar type of persistence faced by the FOMC is considered plausible by Rudebusch (2002). The shocks $\varepsilon_{t}$ are normally distributed and independent of the current and past values of the predictors $\mathbf{x}_{s}$ for all $s \leq t$. Their effect on the latent target rate dies out exponentially over time. This structure allows for temporal dependence in the latent target rate $r_{t}^{*}$ in (3), in addition to the temporal dependence due to autocorrelation in the predictor variables $\mathbf{x}_{t}$. In the empirical application, we compare

\footnotetext{
${ }^{3}$ If we consider $r_{t}^{*}$ as the latent target rate which directs monetary policy, we can follow the economic interpretation of Bernanke and Blinder (1992) and describe $u_{t}$ as an unanticipated monetary policy shock as opposed to the anticipated part captured by $\boldsymbol{\beta}^{\prime} \mathbf{x}_{t}$.
} 
the dynamic ordered probit model with a static model, obtained by setting $\varphi=0$ in (4).

\subsection{Prior specification}

We adopt a Bayesian approach for estimation and inference in the dynamic probit model with endogenous variable selection. Therefore we have to choose a prior for the model parameters in (2)-(4). The prior specifications for most of the parameters are straightforward in the sense that we set them relatively uninformative and conjugate.

For the threshold parameters $\alpha_{j}$ we opt for a flat prior, not imposing any kind of prior asymmetry that would favor a particular target rate adjustment category. However, due to the ordering of the categories of $y_{t}$, we do restrict this prior to the region $\left\{\left(\alpha_{1}, \alpha_{2}\right)^{\prime} \in \mathbb{R}^{2}: \alpha_{1}<\alpha_{2}\right\}$.

Our prior for the autoregressive parameter $\varphi$ is such that stationarity of the $u_{t}$ process is guaranteed. We take a truncated normal distribution: $\varphi \sim \mathcal{N}(b, B) \times$ $\mathbb{I}_{\{\varphi \in S\}}$ with $S=(-1,1)$. We note that for very large $B$ the prior for $\varphi$ becomes a uniform distribution on the region defined by $S$.

The regression parameters deserve some extra attention due to the endogenous variable selection procedure. As noted before, we decompose $\boldsymbol{\beta}$ into the product of inclusion indicators $\boldsymbol{\gamma}=\left(\gamma_{1}, \ldots, \gamma_{K}\right)^{\prime}$ and regression effects $\boldsymbol{\psi}=\left(\psi_{1}, \ldots, \psi_{K}\right)^{\prime}$. We first focus on the inclusion indicators. We take the inclusion of the $k$-th predictor to be a priori independent of the inclusion of all other $K-1$ variables. Consequently, the prior for each $\gamma_{k}$ is a Bernoulli distribution with probability of success $\pi_{k}: \gamma_{k} \mid \pi_{k} \sim$ $\operatorname{Ber}\left(\pi_{k}\right)$. A prior on the $\pi_{k}$ 's implies a prior on the model size. The latter is defined as the number of included covariates given by $N(\gamma)=\sum_{k=1}^{K} \gamma_{k}$. For simplicity we restrict the prior inclusion probabilities to be equal, that is, we set $\pi_{k}=\pi$ for $k=1, \ldots, K$. We opt for a conjugate Beta prior on $\pi$, that is, $\pi \sim \mathcal{B} e\left(c_{1}, c_{2}\right)$. In this setting the prior expected model size is equal to $K c_{1} /\left(c_{1}+c_{2}\right){ }^{4}$ We experiment with different values for the hyperparameters $c_{i}$ to avoid strong prior influence on posterior results. As a starting point we make this prior uninformative in the sense that we consider all values $\pi \in[0,1]$ to be equally likely (which is achieved by setting $\left.c_{1}=c_{2}=1\right)$.

One of the advantages of Kuo and Mallick's (1998) approach is the specification

\footnotetext{
${ }^{4}$ We compute prior expected model size by applying the law of iterated expectations: $\mathrm{E}[N(\gamma)]=$ $\mathrm{E}_{\pi} \mathrm{E}_{\gamma \mid \pi}[N(\gamma)]=\mathrm{E}_{\pi}[K \pi]=\frac{K \cdot c_{1}}{c_{1}+c_{2}}$, because $N(\gamma) \mid \pi \sim \mathcal{B} i n(K, \pi)$.
} 
of the prior for $\psi$. This prior is specified independent of the inclusion indicators $\boldsymbol{\gamma}$, in contrast to the mixture of normals idea of George and McCulloch (1993). This facilitates the posterior simulation considerably and has no undesirable model restrictions. We propose to use a Gaussian prior for $\boldsymbol{\psi}$ with mean a and covariance matrix $\mathbf{A}$, that is, $\boldsymbol{\psi} \sim \mathcal{N}(\mathbf{a}, \mathbf{A})$. As we demonstrate below, in our simulation scheme to obtain posterior results (based on the Gibbs sampler), $\boldsymbol{\psi}$ is sampled conditional on $\gamma$ and vice versa. If $\gamma_{k}=0$, the corresponding $\psi_{k}$ is not identified by the data and is approximately sampled from its prior distribution. This implies that, in order to give predictor $k$ a reasonable chance of getting selected in the next iteration, the prior should generate 'good proposals'. Kuo and Mallick (1998) recommend to make the prior relatively uninformative and choose a diagonal prior covariance matrix $\mathbf{A}$ with elements equal to 16 if the explanatory variables are standardized. We adopt their recommendation, though we have experimented with different values. Provided that these variances do not take extreme values, we find that posterior results are robust.

\subsection{Inference}

We obtain posterior results by using Markov chain Monte Carlo [MCMC] methods, see Tierney (1994) and Robert and Casella (2004). The latent variables $\mathbf{r}^{*}=$ $\left(r_{1}^{*}, \ldots, r_{T}^{*}\right)^{\prime}$ are simulated alongside the model parameters $\boldsymbol{\theta}=\left(\alpha_{1}, \alpha_{2}, \pi, \varphi, \boldsymbol{\psi}^{\prime}, \boldsymbol{\gamma}^{\prime}\right)^{\prime}$, see Tanner and Wong (1987) and Albert and Chib (1993). The posterior density of the parameters and latent variables after having observed a sample $\mathbf{y}=\left(y_{1}, \ldots, y_{T}\right)^{\prime}$ is given by

$$
p\left(\boldsymbol{\theta}, \mathbf{r}^{*} \mid \mathbf{y}\right) \propto p(\boldsymbol{\theta}) p\left(\mathbf{y}, \mathbf{r}^{*} \mid \boldsymbol{\theta}\right),
$$

in which $p(\boldsymbol{\theta})$ and $p\left(\mathbf{y}, \mathbf{r}^{*} \mid \boldsymbol{\theta}\right)$ are the prior density of the model parameters and the complete data likelihood function of the model, respectively. We refer to A for the specific functional forms of these densities.

It is well known that Gibbs sampling in ordered probit models may suffer from mixing difficulties, especially if some form of temporal dependence in the latent variables is allowed, as in (4), see e.g. Liu and Sabatti (2000). We solve this issue partly by fixing the lower threshold $\alpha_{1}$ in (2) at zero and at the same time including an intercept in (3). We only use this reparameterization for posterior simulation. Results are easily converted back into the familiar, interpretable model specification 
for inference. ${ }^{5}$ The MCMC simulation scheme to sample from the posterior is given by:

Step 1. Sample the threshold $\alpha_{2}$ from its full conditional posterior (uniform distribution);

Step 2. Sample $\varphi$ given the other parameters and $\mathbf{r}^{*}$ with a Metropolis-Hastings sampler as suggested by Chib and Greenberg (1994) (proposal values from truncated normal distribution);

Step 3. Sample $\pi$ from its full conditional posterior (Beta distribution);

Step 4. Sample the latent target rate $r_{t}^{*}$ from its full conditional posterior for $t=$ $1, \ldots, T$ (truncated normal distributions);

Step 5. Sample $\gamma_{k}$ from its full conditional posterior for $k=1, \ldots, K$ in a random order, see Kuo and Mallick (1998) (Bernoulli distributions);

Step 6. Sample $\boldsymbol{\psi}$ from its full conditional posterior, see also Kuo and Mallick (1998) (multivariate normal distribution). ${ }^{6}$

The first step proceeds in a similar fashion as for a standard (static) ordered probit model, see e.g. Albert and Chib (1993). Since we do not condition on the first observation, we cannot sample the autoregressive parameter from a truncated normal distribution. Therefore, in Step 2 we implement the Metropolis-Hastings sampler to simulate $\varphi$ as suggested by Chib and Greenberg (1994). In Step 3 the full conditional posterior of $\pi$ reduces to $\pi \mid \boldsymbol{\gamma} \sim \mathcal{B} e\left(c_{1}+N(\boldsymbol{\gamma}), c_{2}+K-N(\boldsymbol{\gamma})\right)$. To take care of the dynamics in the latent variables and the treatment of the first observation, we can rewrite the conditional model and execute the next three steps of the simulation scheme in a straightforward manner. We provide details of our posterior simulation procedure in Appendix A.

\footnotetext{
${ }^{5}$ If we call the intercept $\beta_{0}$, and the new upper threshold $\alpha_{2}^{*}$, then this reparameterization boils down to $\alpha_{1}=-\beta_{0}$ and $\alpha_{2}=\alpha_{2}^{*}-\beta_{0}$. If we set improper priors $\beta_{0} \propto 1$ and $\alpha_{2}^{*} \propto \mathbb{I}_{\left\{\alpha_{2}^{*}>0\right\}}$, the priors in both parameterizations are fully equivalent.

${ }^{6}$ Note that due to the reparameterization, in this step the regression parameters and the intercept are simulated in one block from their joint multivariate normal distribution.
} 


\section{$2.4 \quad$ Forecasting}

To construct real-time Bayesian forecasts of the FOMC target rate decisions that account for model and parameter uncertainty, we have to derive the posterior predictive distributions. The one-step ahead predictive distribution of $y_{s}$ made at time $s-1$ is given by its conditional probability density function

$$
p\left(y_{s} \mid \mathbf{y}^{1, s-1}\right)=\int p\left(y_{s} \mid \boldsymbol{\theta}, \mathbf{r}^{* ; 1, s-1}, \mathbf{y}^{1, s-1}\right) p\left(\boldsymbol{\theta}, \mathbf{r}^{* ; 1, s-1} \mid \mathbf{y}^{1, s-1}\right) d \mathbf{r}^{* ; 1, s-1} d \boldsymbol{\theta},
$$

in which $\mathbf{y}^{n, m}=\left(y_{n}, \ldots, y_{m}\right)^{\prime}$ and $\mathbf{r}^{* ; n, m}=\left(r_{n}^{*}, \ldots, r_{m}^{*}\right)^{\prime},(1 \leq n<m \leq T)$. Given draws from the posterior distribution with $\operatorname{pdf} p\left(\boldsymbol{\theta}, \mathbf{r}^{* ; 1, s-1} \mid \mathbf{y}^{1, s-1}\right)$, we can use Monte Carlo integration to solve the integral in (6). We note that we can analytically compute $p\left(y_{s} \mid \boldsymbol{\theta}, \mathbf{r}^{* ; 1, s-1}, \mathbf{y}^{1, s-1}\right)=p\left(y_{s} \mid \boldsymbol{\theta}, r_{s-1}^{*}\right)$ by evaluations of the normal cumulative distribution function. Because $\boldsymbol{\theta}$ contains the predictor inclusion parameters $\boldsymbol{\gamma}$, each draw from the posterior also indicates a particular selected combination of predictors. According to this principle, forecasts by models which are a posteriori considered more likely get a larger weight in the 'model-free' predictive distribution.

For model evaluation we gauge the predictive power of the model by considering one-step ahead forecasts. We compute these forecasts for the last $T-\tau$ observations, that is, for months $s=\tau+1, \ldots, T$. For every one-step ahead prediction we want to incorporate all sample information revealed by that date. Therefore we update our posterior distribution for every forecast we make.

We limit the computational burden by using importance sampling methods. The forecast for $y_{s}$ is based on all information available up to and including period $s-1$. Hence, based on draws from $p\left(\boldsymbol{\theta}, \mathbf{r}^{* ; 1, s-1} \mid \mathbf{y}^{1, s-1}\right)$ we can construct the forecast for period $s$ according to (6). Before constructing the next one-step ahead forecast, for $y_{s+1}$, we incorporate the extra observed information revealed by the latest target rate decision $y_{s}$ to update the posterior beliefs. We avoid rerunning the entire Gibbs sampler by using importance sampling with the posterior from the previous period, $p\left(\boldsymbol{\theta}, \mathbf{r}^{* ; 1, s-1} \mid \mathbf{y}^{1, s-1}\right)$, as importance function. To construct the forecast for $y_{s+1}$ we actually need a sample from $p\left(\boldsymbol{\theta}, \mathbf{r}^{* ; 1, s} \mid \mathbf{y}^{1, s}\right)$, but our importance function does not provide a draw for $r_{s}^{*}$. We solve this issue by extending the posterior from the previous period with $p\left(r_{s}^{*} \mid \boldsymbol{\theta}, \mathbf{r}^{* ; 1, s-1}, \mathbf{y}^{1, s}\right)$ and, hence, we obtain $r_{s}^{*}$ by simulating from a truncated normal distribution. In Appendix B we describe this forecasting procedure in full detail and show how to derive the importance weights. 


\section{Data and target rate characteristics}

We investigate the federal funds target rate at a monthly frequency for the period from January 1990 till June 2008. It can be argued that during this sample period, covering most of Greenspan's term as chairman of the Board of Governors plus the start of Bernanke's reign, the Federal Reserve's monetary policy objectives have been kept constant. ${ }^{7}$ We build on this by assuming that the macroeconomic and financial variables that are most closely monitored by the FOMC (and thus are most informative for predicting target rate changes) have been the same throughout this period. Put differently, we assume that structural breaks in the parameters of the ordered probit model in (2)-(4) do not occur.

The FOMC meets eight times per year at previously set dates. Our sample period, which covers 222 months in total, contains 148 months with such a scheduled meeting. In addition, unscheduled meetings of the FOMC are held occasionally (sometimes by conference call), if required by sudden unexpected economic developments or other major events affecting the economy. In addition to the 148 months with scheduled meetings, we observe nine more months with unscheduled meetings in our sample. ${ }^{8}$ We argue that the decision making process during these unscheduled meetings is no different from the regular meetings. The only difference is that $\mathbf{x}_{t}$ takes atypical values that urge an extra meeting, but given these exogenous variables, the way the outcome is decided upon (i.e., the model parameters) is identical. ${ }^{9}$

\footnotetext{
${ }^{7}$ During our sample period, substantial changes did occur in Federal Reserve's operating procedures and communication policy (see Poole; 2005; Ehrmann and Fratzscher; 2007, among others).

${ }^{8}$ Specifically, these months cover decisions made on January 9, 1991; April 30, 1991; 13 September, 1991; April 9, 1992; September 4, 1992; October 15, 1998; April 18, 2001; and September 17, 2001 (target rate decreases) and on April 18, 1994 (target rate increase). There are also months with both unscheduled and scheduled meetings. For example, an unscheduled target rate decrease was announced on January 22, 2008, but this was followed later that month by another target rate cut following the scheduled meeting on January 29-30. We typify such months as months with a scheduled meeting and therefore include them among the 148 'scheduled' months.

${ }^{9}$ We have examined an alternative definition of the dependent variable $y_{t}$ in (1), which has observations if and only if there is a scheduled meeting in month $t$. Months without an unscheduled meeting are not omitted from the sample, but $y_{t}$ is treated as missing in such a case. Estimation results for this specification are not much different from those reported in Section 4. In particular, similar predictor variables are considered relevant in forecasting the target rate decisions and estimated thresholds do not indicate substantial differences. Hence, removing the unscheduled meetings from the data set would mean throwing away information while we already have relatively few observations.
} 
Two remarks are in order. First, as discussed in the previous section, our dynamic probit model is specified in calendar time. Hence, about one third of our sample period concerns months without an FOMC meeting. In this case, $y_{t}$ as defined in (2) is not observed and the only thing we know is how the unobserved target rate behaves: $r_{t}^{*} \mid \boldsymbol{\theta}, r_{t-1}^{*} \sim \mathcal{N}\left(\boldsymbol{\beta}^{\prime} \mathbf{x}_{t}+\varphi\left(r_{t-1}^{*}-\boldsymbol{\beta}^{\prime} \mathbf{x}_{t-1}\right), 1\right)$, according to (3). Hence, such a month does not provide direct information about the unknown model parameters. We note that this is different from months with an FOMC meeting at which it is decided to keep the target unchanged. According to the model specification in (2), in that case the difference between the latent target rate $r_{t}^{*}$ and the prevailing actual target $r_{t-1}$ rate must have realized in the range $\left(\alpha_{1}, \alpha_{2}\right]$ and this truncates $r_{t}^{*}$.

Second, because we do not exclude months without meetings, neither in the model estimation, nor in the forecasting experiment, our model is makes monthly predictions. Hence, due to our sample selection, every forecast we construct is necessarily conditioned on the event of a meeting and each forecasting statement should start with saying: "If the FOMC organized a meeting next month, the forecast for its outcome would be ...".

Table 1: Summary statistics

\begin{tabular}{lllll}
\hline \hline \multicolumn{2}{c}{ FOMC decisions } & & \multicolumn{2}{c}{ Federal funds target rate } \\
\# Decreases & 40 & & Mean & 4.35 \\
\# No-changes & 86 & & Minimum (June 2003 - May 2004) & 1.00 \\
\# Increases & 31 & & Maximum (January 1990 - June 1990) & 8.25 \\
& & Standard deviation & 1.85 \\
\hline \hline
\end{tabular}

The announced target rates are displayed Figure 1, with summary statistics being provided in Table 1. Over the sample period the target rate varied considerably, between a minimum of 1.00 percent in 2003-4 and a maximum of 8.25 percent during the first half of 1990. The circles in Figure 1 indicate the decisions to change the target rate made by the FOMC. We represent no-change outcomes by diamonds. 
With 40 months with a decrease and 31 months with an increase, the FOMC seems slightly more reluctant to set a higher target rate, although we have to correct for the effects of the covariates. Figure 1 clearly shows that decisions of the same type appear in clusters. For example, periods of sustained declines of the target rate occurred in the early nineties, in 2001, and during the final year of the sample period. To a large extent these target rate declines coincide with U.S. recessions as declared by the NBER, as shown in Figure 1 by the shaded areas. Similarly, multiple consecutive decisions to increase the target were made during 1994, 1999, and the period from mid-2004 till mid-2006. These clusters can be explained by our model via two mechanisms. First, it may be due to temporal dependence in the predictor variables and, second, conditional on these covariates there is the persistence in the decisions caused by interest rate smoothing.

Figure 1: Federal funds target rate and FOMC decisions

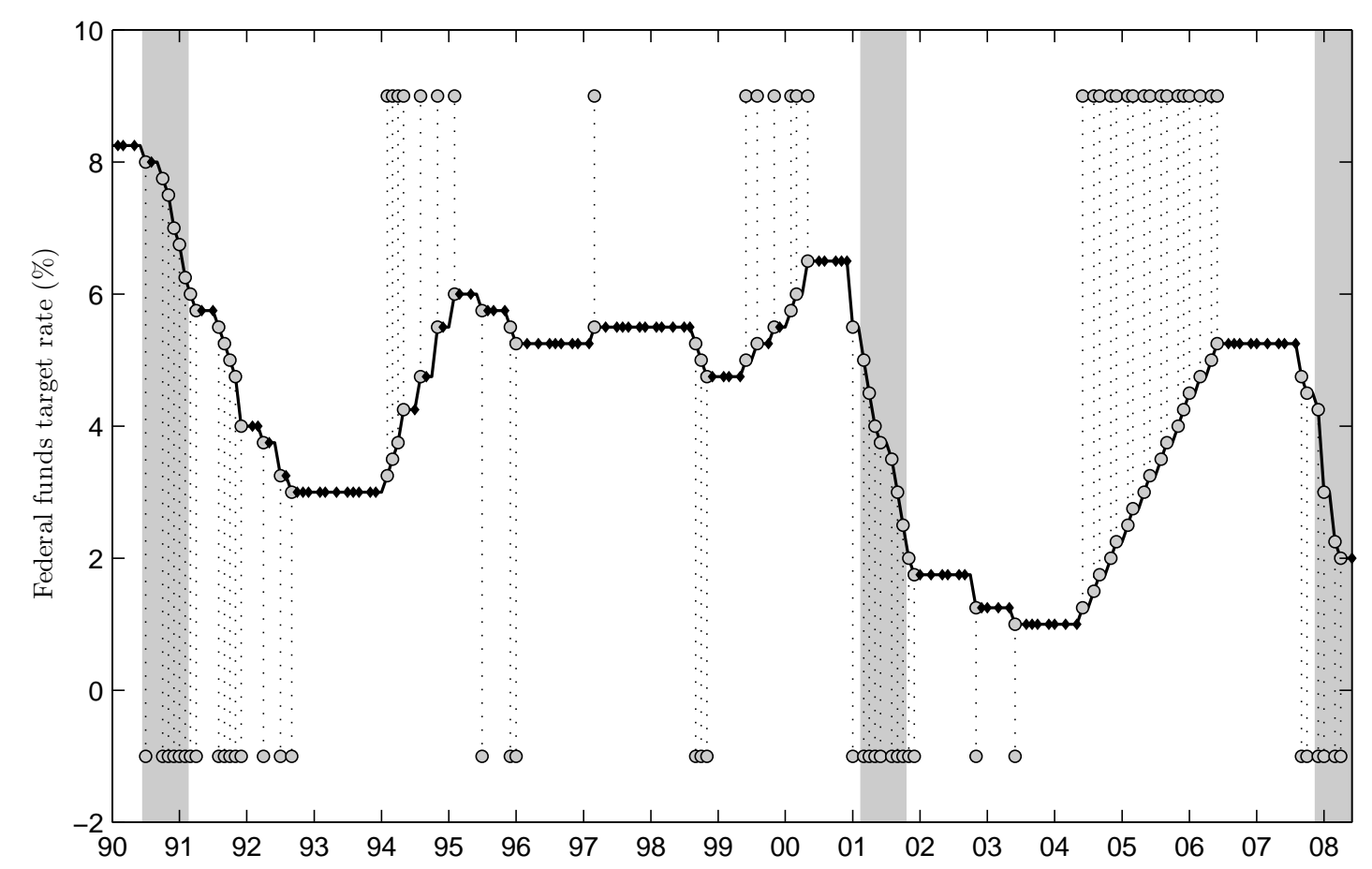

Our data set further consists of a set of macroeconomic and financial variables that are considered potential predictors for the FOMC target rate decisions. These variables can be categorized in three groups. The first group comprises measures related to inflation, output and (un)employment. These variables are most closely related to the monetary policy objectives of the Federal Reserve and for this rea- 
son might be expected to play a key role in the FOMC decision making process. The second group of variables consists of recent realizations of several other macro and financial variables that provide information on economic developments. Most of the variables in this group, such as new orders and building permits, have a forward-looking character. As discussed in the introduction, the FOMC considers multiple economic indicators in its deliberations, reflecting developments in financial markets, the labor market, and the housing market, among others. Including this second group of variables allows us to examine whether these indicators provide any supplemental information for predicting target rate decisions, in addition to inflation and output measures. The third group of variables consists of survey-based measures of consumer confidence and forecasts of inflation, output and interest rates. The latter are taken from the Survey of Professional Forecasters conducted by the Federal Reserve Bank of Philadelphia, see Croushore (1993) for a description of the way these forecasts are constructed. Including this group of variables is motivated, among others, by Ang et al. (2007) and Campbell and Diebold (2009), who demonstrate that survey-based measures provide more accurate out-of-sample forecasts for inflation and stock returns than historical macroeconomic and financial variables.

A relevant feature for the macroeconomic variables in the first and second group of predictors is that they are subject to revisions after their initial release. As a consequence, the currently available time series is different from the one that was at the FOMC's disposal at the time they met. These revisions can be substantial, in particular for output and employment related variables. Diebold and Rudebusch (1991) and Stark and Croushore (2002), among others, analyze the consequences and potential pitfalls in case a forecasting experiment is conducted with latest-available data instead of variables measured in real time, see also Croushore (2006) for a recent survey. Note that the survey-based measures in the third group of predictors are not subject to revisions, which can be considered an additional advantage of this type of variables. In order to make our empirical analysis as realistic as possible, we employ data as available on a real-time basis. For this purpose, we combine data from the ALFRED database of the Federal Reserve Bank of St. Louis, the Federal Reserve Bank of Philadelphia's real-time database and real-time data for the components of The Conference Board Leading Economic Indicator. ${ }^{10}$ Our resulting data set is set

\footnotetext{
${ }^{10}$ The real-time database of the Federal Reserve Bank of Philadelphia is constructed and maintained as described in Croushore and Stark (2001). We use their (monthly) vintages of GDP, but due to some redefinitons we encounter problems in forming a real-time output gap. Therefore we
} 
up such that the vector of covariates $\mathbf{x}_{t}$ contains potentially relevant information for the target rate decision in month $t$, as it was historically available at the end of month $t-1$, when the forecast for the coming month was made.

Table 2 lists the complete set of 33 potential predictors we use in our analysis. All variables are transformed to stationarity, mostly by converting them to growth rates; see the final column of Table 2. Two further issues are worth mentioning. First, variables measured only at a quarterly frequency, such as the SPF forecasts, are transformed to monthly observations by simply keeping the value constant for the three months within a quarter. We justify this by observing that during this threemonth period no new information about this variable is revealed. Second, we allow for a so-called 'averaging' period. By this we mean that we take moving averages or growth rates over the $m$ most recent available observations of the predictors. This reflects the idea that possibly the FOMC does not (only) focus on what has happened to a certain economic indicator in the most recent month, which can be a noisy signal, but instead (or also) considers developments over a longer period of a few months. We experiment with different values for $m$ to analyze the robustness of our results with respect to the choice of $m$.

\section{Empirical results}

This section is divided into three parts. First, we consider estimation results for the dynamic (and static) ordered probit model when using the full sample period from January 1990 till June 2008, focusing on the question which variables appear to be most informative for FOMC target rate decisions. Second, we present real-time out-of-sample forecasts of the target rate decisions during the period from January 2001 till June 2008. Third and finally, we discuss a number of robustness checks that examine the sensitivity of the results to the choice of priors and the transformations applied to the predictor variables.

construct our own series of real potential GDP in real time by applying an HP-filter per vintage to real-time GDP. 
Table 2: Set of potential predictors

\begin{tabular}{|c|c|c|c|c|}
\hline \multirow[t]{2}{*}{ Variable } & \multirow[t]{2}{*}{ Acronym } & \multicolumn{2}{|c|}{$\operatorname{Pr}\left[\gamma_{k}=1 \mid \mathbf{y}\right]$} & \multirow[t]{2}{*}{ Transf. } \\
\hline & & Dynamic & Static & \\
\hline \multicolumn{5}{|l|}{ Panel A: Monetary policy variables } \\
\hline 1. Inflation, CPI: U.S. city average: All items: Seasonally adjusted & INF & 0.023 & 0.187 & $g r$ \\
\hline 2. Output gap, Real GDP less its HP-filtered trend & OUT & 0.474 & 0.166 & $a v$ \\
\hline 3. Unemployment gap, Unemployment rate less CBO NAIRU & UG & 0.054 & 0.038 & $a v$ \\
\hline \multicolumn{5}{|l|}{ Panel B: Other macroecnomic and financial variables } \\
\hline 4. TCB LEI, The Conference Board's leading economic indicator & LEI & 0.017 & 0.012 & $g r$ \\
\hline 5. BCI-01 Average weekly hours, manufacturing & WHM & 0.049 & 0.020 & $g r$ \\
\hline 6. BCI-05 Average weekly initial claims for unemployment insurance & CUI & 0.014 & 0.010 & $g r$ \\
\hline 7. BCI-08 Manufacturers new orders, consumer goods and materials & NOC & 0.017 & 0.014 & $g r$ \\
\hline 8. BCI-32 Vendor performance, slower deliveries diffusion index & VPI & 0.016 & 0.022 & $g r$ \\
\hline 9. BCI-27 Manufacturers new orders, nondefense capital goods & NOK & 0.012 & 0.008 & $g r$ \\
\hline 10. BCI-29 Building permits, new private housing units & NBP & 0.039 & 0.009 & $g r$ \\
\hline 11. BCI-19 Stock prices, 500 common stocks & SP5 & 0.009 & 0.010 & $g r$ \\
\hline 12. BCI-106 Money supply, M2 & M2 & 0.009 & 0.008 & $g r$ \\
\hline 13. BCI-129 Interest rate spread, 10-y Treasury bonds less Fed funds & $10 \mathrm{TFF}$ & 0.052 & 0.969 & $a v$ \\
\hline 14. TCB CCI, The Conference Board's coincident index & CCI & 0.336 & 0.020 & $g r$ \\
\hline 15. BCI-41 Employees on nonagricultural payrolls & ENP & 0.035 & 0.013 & $g r$ \\
\hline 16. BCI-51 Personal income less transfer payments & PI & 0.066 & 0.016 & $g r$ \\
\hline 17. BCI-47 Index of industrial production & IP & 0.481 & 0.412 & $g r$ \\
\hline 18. BCI-57 Manufacturing and trade sales & MTS & 0.010 & 0.017 & $g r$ \\
\hline 19. Interest rate spread, 6-month T-bill less Fed funds & $6 \mathrm{TFF}$ & 1.000 & 1.000 & $a v$ \\
\hline 20. Capacity utilization: Manufacturing & CUM & 0.065 & 0.043 & $a v$ \\
\hline 21. Household sector: Liabilites: Household credit market debt outstanding & $\mathrm{HCM}$ & 0.109 & 0.064 & $g r$ \\
\hline 22. Total consumer credit outstanding & $\mathrm{TCC}$ & 0.023 & 0.013 & $g r$ \\
\hline \multicolumn{5}{|l|}{ Panel C: Survey measures and professional forecasts } \\
\hline 23. TCB Consumer confidence & $\mathrm{CC}$ & 0.083 & 0.144 & $a v$ \\
\hline 24. TCB Consumer confidence: Present situation & $\mathrm{CCP}$ & 0.518 & 0.131 & $a v$ \\
\hline 25. TCB Consumer confidence: Expectations & $\mathrm{CCE}$ & 0.036 & 0.032 & $a v$ \\
\hline 26. Univ of Michigan consumer sentiment index & CS & 0.034 & 0.092 & $a v$ \\
\hline 27. Univ of Michigan consumer sentiment index: Current conditions & $\mathrm{CSC}$ & 0.038 & 0.589 & $a v$ \\
\hline 28. Univ of Michigan consumer sentiment index: Expectations & CSE & 0.030 & 0.052 & $a v$ \\
\hline 29. Average (mean) duration of unemployment & $\mathrm{MDU}$ & 0.275 & 0.174 & $a v$ \\
\hline 30. ISM Purchasing managers index & ISM & 0.019 & 0.109 & $a v$ \\
\hline 31. Anxious index: Probability of decline in real GDP in the following quarter & AI & 0.021 & 0.016 & $a v$ \\
\hline 32. SPF: Mean expected 3-month T-bill rate in the following 4 quarters & $3 \mathrm{TE}$ & 0.142 & 0.966 & $a v$ \\
\hline 33. SPF: Mean expected CPI inflation rate in the following 4 quarters & INE & 0.052 & 0.034 & $a v$ \\
\hline
\end{tabular}

Note: We use two types of transformations for the predictor variables. Dependent on the behavior and character of the explantory variable we compute the annualized growth rate $\frac{1200}{m} \frac{\Delta_{m} x_{t}}{L^{m} x_{t}}(g r)$ or a moving average $\frac{1}{m}\left(1+L+\ldots+L^{m-1}\right) x_{t}$ (av) over the $m$ most recent months. 


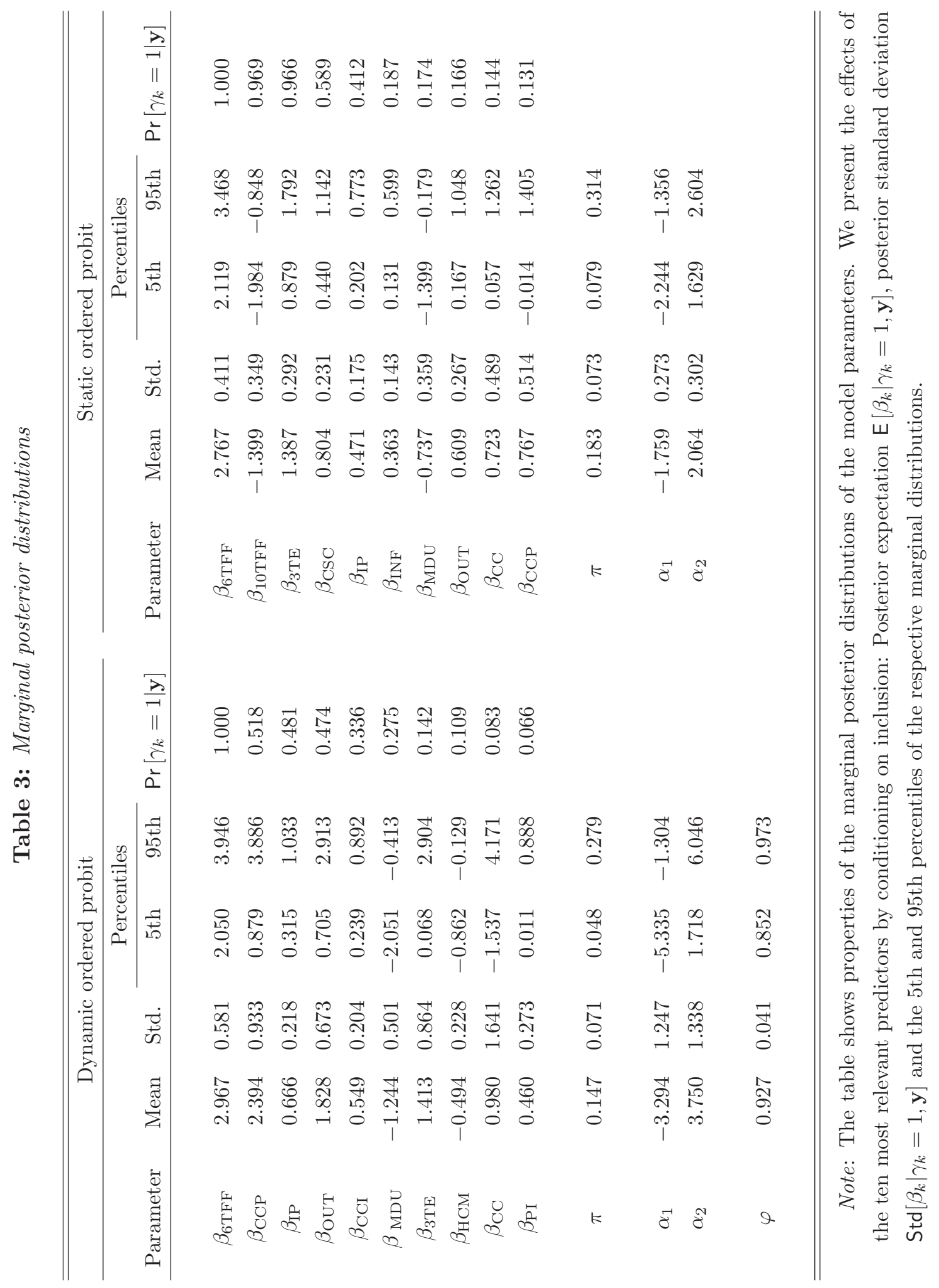




\subsection{Estimation results}

We present the key properties of the marginal posterior distributions of the dynamic ordered probit model parameters in the left panel of Table 3. We obtain these results by applying the MCMC simulation scheme discussed in Section 2.3 using the full sample period from January 1990 till June 2008 with an uninformative $\mathcal{B} e(1,1)$ prior on the predictor inclusion probability $\pi$ and an averaging period equal to one month $(m=1)$. For comparison, we present the posterior results for the nested static ordered probit model, that is, with the restriction $\varphi=0$ in (4), in the right panel of this table.

Several conclusions emerge from these posterior results. First, we find strong evidence for temporal dependence in the target rate decisions that goes beyond the dependence caused by the inclusion of autocorrelated predictor variables. The posterior mean of the autoregressive parameter in the $\operatorname{AR}(1)$ specification for the error process $u_{t}$ in the dynamic probit model is equal to 0.93 . Since the posterior mass is tightly concentrated around values close to 0.9 , the effect of a shock $\varepsilon_{t}$ remains noticeable for a substantial period of time. The posterior mean of 0.93 , for example, corresponds with a half-life of shocks of ten months.

Second, based on the marginal posterior inclusion probabilities $\operatorname{Pr}\left[\gamma_{k}=1 \mid \mathbf{y}\right]$, we observe that a limited number of predictor variables appears to be informative for the target rate decisions. We show these probabilities for the ten most frequently sampled covariates in the rightmost columns of the panels in Table 3, together with summary statistics of the posterior for the average inclusion probability $\pi .{ }^{11}$ For the dynamic model we find that six variables have a conditional posterior inclusion probability that exceeds the posterior mean $\mathrm{E}[\pi \mid \mathbf{y}]$, which equals 0.15 . These variables are the spread between the six-month T-bill rate and the effective federal funds rate (6TFF), The Conference Board's consumer confidence index: present situation (CCP), industrial production growth (IP), the output gap (OUT), The Conference Board's coincident index (CCI) and the expected mean duration of unemployment (MDU). The spread between the six-month T-bill rate and the effective federal funds rate $(6 \mathrm{TFF})$ is by far the most important predictor, in agreement with Hamilton and Jordà (2002). This spread is included in the model with probability equal to one, which furthermore is almost twice as high as the inclusion probability of the second-most frequently sampled variable, being The Conference Board's consumer

\footnotetext{
${ }^{11}$ We display the marginal posterior inclusion probabilities for all examined predictors in Table 2.
} 
confidence measure of present economic conditions. Although its inclusion probability of 0.14 is slightly smaller than the posterior of $\pi$, the SPF forecast of the three-month T-bill rate (3TE) also contains relevant predictive content. Interestingly, inflation only has a posterior inclusion probability of 0.023 , indicating that lagged inflation is not important for predicting FOMC decisions.

For the static model we also find six variables for which $\operatorname{Pr}\left[\gamma_{k}=1 \mid \mathbf{y}\right]>\mathbf{E}[\pi \mid \mathbf{y}]=$ 0.18. These include the spread between the six-month T-bill and the effective federal funds rate (6TFF), growth in industrial production (IP) and the professional forecast of the three-month T-bill rate, which also are found to be important in the dynamic model. In addition, the spread between the ten-year T-bond rate and the effective federal funds rate (10TFF), the University of Michigan's consumer sentiment index: current conditions (CSC), and the inflation rate (INF) satisfy this condition. Although their inclusion probabilities are strictly below the posterior mean of $\pi$, the output gap (OUT), expected mean duration of unemployment (MDU) and The Conference Board's consumer confidence measures (CC, CCP) contribute substantially to describing FOMC decisions.

It is also relevant to note that for both models the posterior mean of the inclusion probability $\pi$ is considerably smaller than its prior mean, which is equal to 0.5. Hence, the data indicate a much smaller expected model size than suggested by the prior distribution of $\pi$. Furthermore, the posterior inclusion probabilities are lower for the dynamic probit model, which is due to the strong explanatory power of the autoregressive dynamics in this model. In Section 4.3 we discuss other Beta priors for $\pi$ to check the robustness of our results.

If we exclusively focus on the marginal inclusion properties of the predictors, the potential interaction between covariates remains hidden, as pointed out by Doppelhofer and Weeks (2009a). In order to investigate these interactions we compute Doppelhofer and Weeks' (2009a) jointness measure for all pairs of explanatory variables $\left\{x_{k t}, x_{l t}\right\},(k \neq l=1, \ldots, K) .{ }^{12}$ This measure, denoted $J_{k, l}$, accounts for both joint inclusion and exclusion of the two variables over the total model space to assess their mutual dependence. Negative values indicate that the two variables are substitutes, which means that they contain approximately the same information content with respect to the target rate decisions. Positive values suggest a complementary pair such that the two variables are jointly in/excluded most of the time and to-

\footnotetext{
${ }^{12}$ There are alternatives to this measure, see the comments in Doppelhofer and Weeks (2009b).
} 
gether they are more informative than in isolation. If $J_{k, l}=0$, inclusion of variable $k$ is a posteriori independent of variable $l$ 's inclusion.

Table 4: Posterior jointness measures for predictor pairs

\begin{tabular}{|c|c|c|c|c|c|c|c|}
\hline \multicolumn{4}{|c|}{ Dynamic ordered probit } & \multicolumn{4}{|c|}{ Static ordered probit } \\
\hline \multicolumn{2}{|c|}{ Complements } & \multicolumn{2}{|c|}{ Substitutes } & \multicolumn{2}{|c|}{ Complements } & \multicolumn{2}{|c|}{ Substitutes } \\
\hline Pair $\{k, l\}$ & $J_{k, l}$ & Pair $\{k, l\}$ & $J_{k, l}$ & Pair $\{k, l\}$ & $J_{k, l}$ & Pair $\{k, l\}$ & $J_{k, l}$ \\
\hline $\mathrm{CC}$ CCE & 2.57 & CCI IP & -3.19 & CSC 3TE & 2.96 & 10TFF OUT & -3.43 \\
\hline MDU OUT & 1.91 & CCP OUT & -2.67 & 10TFF CSC & 2.64 & CC CSC & -2.69 \\
\hline CS CSE & 1.64 & CCI PI & -1.87 & CS CSE & 2.55 & CSC OUT & -2.47 \\
\hline INF PI & 1.58 & CCP MDU & -1.55 & MDU OUT & 2.30 & CCP CSC & -2.44 \\
\hline $3 \mathrm{TE}$ INE & 1.51 & WHM PI & -1.32 & CUM MDU & 1.94 & CS CSC & -2.06 \\
\hline INF CSC & 1.43 & CCI HCM & -1.24 & IP MDU & 1.65 & CCE 3TE & -1.89 \\
\hline CCI ENP & 1.39 & NOC IP & -1.00 & CCE CUM & 1.59 & 10TFF INE & -1.81 \\
\hline NOK HCM & 1.36 & $\mathrm{CC}$ CCP & -0.92 & INE OUT & 1.49 & 10TFF CCP & -1.74 \\
\hline WHM OUT & 1.29 & WHM IP & -0.91 & CCP 3TE & 1.37 & CSC CSE & -1.62 \\
\hline PI MTS & 1.22 & CCP HCM & -0.82 & IP CCP & 1.32 & $\mathrm{CSC} \mathrm{MDU}$ & -1.58 \\
\hline
\end{tabular}

Note: The table shows Doppelhofer and Weeks' (2009a) jointness measure for predictor pairs in the dynamic and static ordered probit models estimated for the full-sample period January 1990 - June 2008, using a $\mathcal{B} e(1,1)$ prior for the variable inclusion probability $\pi$ and an averaging period $m=1$ for the predictor variables. The jointness measure is defined as $J_{k, l}=\log [q(k, l) q(\bar{k}, \bar{l})]-\log [q(k, \bar{l}) q(\bar{k}, l)]$ in which $q(k, l)=\operatorname{Pr}\left[\gamma_{k}=1, \gamma_{l}=1 \mid \mathbf{y}\right]$ is the posterior joint inclusion probability, $q(\bar{k}, \bar{l})=\operatorname{Pr}\left[\gamma_{k}=0, \gamma_{l}=0 \mid \mathbf{y}\right]$ the posterior joint exclusion probability and $q(\bar{k}, l)=\operatorname{Pr}\left[\gamma_{k}=0, \gamma_{l}=1 \mid \mathbf{y}\right]$ and $q(k, \bar{l})=\operatorname{Pr}\left[\gamma_{k}=1, \gamma_{l}=0 \mid \mathbf{y}\right]$ are the two posterior mutual exclusion probabilities. Jeffreys' classification can be used to assess significance: $\left|J_{k, l}\right|>2$, strong relation; $1<\left|J_{k, l}\right|<2$, significantly related; $\left|J_{k, l}\right|<1$, no significant relation. Negative values indicate substitutes, positive values signal a complementary relation.

Table 4 shows the ten pairs of strongest complements and substitutes for both the dynamic (left panel) and static (right panel) ordered probit models. The TCB consumer confidence index and its expectations component are strong complements. We find a similar result for the consumer sentiment indices by the University of Michigan (CS and CSE); measures of the current economic situation need the forward-looking character of consumers' future expectations to jointly approximate the present situation and predict FOMC decisions. Many of the complementary pairs contain a current economic activity measure and a type of forward-looking variable. For example, the output gap and the expected duration of unemployment, which both are important predictors when considered in isolation as shown in Table 3, are often jointly in/excluded. Growth in TCB's coincident index is a strong substitute 
for both industrial production and personal income. This may not be surprising, given that these variables are two of the four constituents of the CCI (together with employment and manufacturing sales). The pairs of substitutes mostly consist of current economic activity measures, for example, present consumer confidence and the output gap or hours of manufacturing and personal income.

For the static probit model we find more predictor pairs that form strong substitutes. Although in this specification the expected model size is larger, many of the different included variables contain the same information for the target rate decisions. Especially pairs of economic activity variables and different present consumer confidence measures contain similar predictive content. We observe that the SPF forecast of the three-month T-bill rate is a strong complement for current consumer sentiment $\left(J_{\mathrm{CSC}, 3 \mathrm{TE}} \approx 3\right)$. The latter, in turn, forms a strong complementary pair with the spread between the ten-year T-bond and the federal funds rate (10TFF); the pair has a jointness measure of 2.6. With respect to the significant complementary pairs, we find a similar result as in the dynamic model: activity variables and forward-looking measures mutually complement each other.

In Table 5 we provide further information about the selected predictor combinations. We show the ten posterior most likely combinations of covariates. Obviously these models are mostly combinations of the posterior likely predictors discussed above. As mentioned before, the autoregressive component in the dynamic probit model apparently accounts for a large part of the dynamics in the latent target rate $r_{t}^{*}$. The static ordered probit specification needs more explanatory variables to capture the dynamics in the target rate, as demonstrated here by the larger posterior most likely combinations of covariates. We expect the main predictors which are important in the static model but less in the dynamic variant (10TFF, 3TE, CSC and IP) to pick up the strong dynamics in $r_{t-1}^{*}$, because, except the growth of industrial production, they show strong persistence. We find that these four covariates jointly explain about 50 percent of the total variation in the lagged latent target rate.

For the dynamic probit model, the model that includes the interest rate spread (6TFF), the present situation consumer confidence (CCP) and TCB's coincident index (CCI) is the most likely combination with a posterior probability of 0.07 . The second most likely model substitutes industrial production for CCI. We have seen before that CCI and IP form a strong pair of substitutes. Most of the included variables measure a type of current economic activity, such as industrial production, coincident index or the output gap. Survey measures and professional forecasts 
Table 5: Posterior most likely combinations of predictors

\begin{tabular}{|c|c|c|c|c|c|c|c|c|c|c|}
\hline \multicolumn{10}{|c|}{ Model $M_{i}$} & \multirow[t]{2}{*}{ Probability } \\
\hline \multicolumn{10}{|c|}{ Panel A: Dynamic probit model } & \\
\hline $6 \mathrm{TFF}$ & & & $\mathrm{CCP}$ & & & CCI & & & & 0.067 \\
\hline $6 \mathrm{TFF}$ & IP & & $\mathrm{CCP}$ & & & & & & & 0.061 \\
\hline $6 \mathrm{TFF}$ & & & $\mathrm{CCP}$ & & & & & & & 0.056 \\
\hline $6 \mathrm{TFF}$ & IP & OUT & & & & & & & & 0.042 \\
\hline $6 \mathrm{TFF}$ & IP & OUT & & $\mathrm{MDU}$ & & & & & & 0.030 \\
\hline $6 \mathrm{TFF}$ & & OUT & & & & CCI & & & & 0.023 \\
\hline $6 \mathrm{TFF}$ & & OUT & & $\mathrm{MDU}$ & & $\mathrm{CCI}$ & & & & 0.023 \\
\hline $6 \mathrm{TFF}$ & IP & & & & & & & & & 0.011 \\
\hline $6 \mathrm{TFF}$ & & OUT & & & $3 \mathrm{TE}$ & & & & & 0.011 \\
\hline $6 \mathrm{TFF}$ & IP & & & & & & & & & 0.010 \\
\hline \multicolumn{11}{|c|}{ Panel B: Static probit model } \\
\hline $6 \mathrm{TFF}$ & & & & & $3 \mathrm{TE}$ & $10 \mathrm{TFF}$ & & $\mathrm{CSC}$ & & 0.191 \\
\hline $6 \mathrm{TFF}$ & & & & & $3 \mathrm{TE}$ & $10 \mathrm{TFF}$ & & $\mathrm{CSC}$ & INF & 0.070 \\
\hline $6 \mathrm{TFF}$ & IP & & & & $3 \mathrm{TE}$ & $10 \mathrm{TFF}$ & & $\mathrm{CSC}$ & & 0.065 \\
\hline $6 \mathrm{TFF}$ & IP & & $\mathrm{CCP}$ & & $3 \mathrm{TE}$ & $10 \mathrm{TFF}$ & & & & 0.038 \\
\hline $6 \mathrm{TFF}$ & IP & & & & $3 \mathrm{TE}$ & $10 \mathrm{TFF}$ & & & & 0.025 \\
\hline $6 \mathrm{TFF}$ & & & & & $3 \mathrm{TE}$ & $10 \mathrm{TFF}$ & ISM & $\mathrm{CSC}$ & & 0.025 \\
\hline $6 \mathrm{TFF}$ & IP & OUT & & $\mathrm{MDU}$ & $3 \mathrm{TE}$ & $10 \mathrm{TFF}$ & & & & 0.019 \\
\hline $6 \mathrm{TFF}$ & & & & & $3 \mathrm{TE}$ & $10 \mathrm{TFF}$ & & & & 0.012 \\
\hline $6 \mathrm{TFF}$ & IP & & & $\mathrm{MDU}$ & $3 \mathrm{TE}$ & $10 \mathrm{TFF}$ & & $\mathrm{CSC}$ & & 0.011 \\
\hline $6 \mathrm{TFF}$ & IP & & & & $3 \mathrm{TE}$ & $10 \mathrm{TFF}$ & & $\mathrm{CSC}$ & INF & 0.011 \\
\hline
\end{tabular}

Note: The table shows the posterior most likely combinations of predictor variables in the dynamic ordered probit model (Panel A) and static ordered probit model (Panel B) estimated for the full-sample period January 1990 - June 2008, using a $\mathcal{B} e(1,1)$ prior for the variable inclusion probability $\pi$ and an averaging period $m=1$ for the predictor variables. The posterior model probability of combination $M_{i}=\left(\gamma_{i, 1}, \ldots, \gamma_{i, K}\right)^{\prime}, \gamma_{i, k} \in\{0,1\},\left(i=1,2, \ldots, 2^{K}, k=1, \ldots, K\right)$, is computed by counting the number of occurrences of this particular combination in the simulation sample of the posterior distribution. 
complement these combinations. The SPF three-month T-bill rate forecast, consumer confidence indexes and households expectations about the duration of unemployment are selected most often. The probabilities of individual models are rather small, indicating that model uncertainty is substantial. Consequently, averaging over models for descriptive or forecasting purposes may be preferable compared to relying upon a single specification with a particular choice of explanatory variables.

We measure the effects of the covariates on the target rate decisions by the marginal posteriors of the parameters $\beta_{k}$. We focus on the effect of an explanatory variable conditional on being included in the model. We therefore consider the properties of the marginal conditional densities $p\left(\beta_{k} \mid \gamma_{k}=1, \mathbf{y}\right)$ as reported in Table 3 . Obviously, we can only draw meaningful conclusions about these effects if the specific variable is incorporated frequently enough, that is, if $\operatorname{Pr}\left[\gamma_{k}=1 \mid \mathbf{y}\right]$ is reasonably large. Otherwise its effect is only weakly identified by the data and conclusions would be based on a small sample from the prior of $\psi_{k}$. Table 3 shows the mean, standard deviation and the 5 th and 95th percentiles of the posterior distributions conditional on predictor inclusion. We can compute the unconditional posterior mean of $\beta_{k}$ by using $\mathrm{E}\left[\beta_{k} \mid \mathbf{y}\right]=\mathrm{E}\left[\beta_{k} \mid \gamma_{k}=1, \mathbf{y}\right] \cdot \operatorname{Pr}\left[\gamma_{k}=1 \mid \mathbf{y}\right]$, because $\mathrm{E}\left[\beta_{k} \mid \gamma_{k}=0, \mathbf{y}\right]=0$.

Economic activity measures all have a positive effect, that is, larger values of these variables imply a higher likelihood of a target rate increase. This corresponds well with the idea that the FOMC tries to temper economic activity during expansionary periods by setting a higher target rate, in order to prevent inflation from becoming too high. Households expecting that it will become more difficult to find a job in the coming months indicates a slowdown in economic growth. The likelihood that the Fed will intervene and stimulate the economy by setting a lower target rate will increase, which explains why $\operatorname{Pr}\left[\beta_{\mathrm{MDU}}<0 \mid \gamma_{\mathrm{MDU}}=1, \mathbf{y}\right] \approx 1$. A similar explanation holds for the effect of growth in the households debt outstanding (HCM).

The negative effect of the spread between the ten-year T-bond rate and the effective federal funds rate in the static probit model may seem strange at first sight. However, we can interpret this variable as a proxy for long-run inflation expectations, as pointed out in, e.g., Estrella and Mishkin (1997). As such, it may not be a variable the FOMC reacts upon directly, but it rather measures market expectations of what the FOMC will decide at upcoming meetings and what the long run impact of its decisions will be. If people expect inflation to be high for a relatively long period of time as the result of a relatively low target rate, long-term interest rates should increase to compensate. As such, the ten-year T-bond rate may be more a predictor 
of FOMC outcomes than an indicator to which the FOMC reacts. This reasoning is contrary to the explanation of the effect of a short-term spread like 6TFF, which represents short-term market expectations about inflation/economic activity to which the FOMC does react. Estrella and Mishkin (1997) also find different predictive natures of spreads with different maturities with regard to forecasting inflation and output growth. Mishkin (1990a,b) discusses similar issues as well.

To get a better understanding of how strong the effects of the explanatory variables are, we display the three category probabilities as functions of a predictor value in Figures 2 and 3 for the dynamic and static probit models, respectively. We show effects of the six posterior most relevant predictors. The steeper the graphs are, the stronger the effect of the explanatory variable under consideration. We see that $6 \mathrm{TFF}, \mathrm{CCP}$ and OUT have the largest impact on the type of FOMC decision. In Figure 3 we observe that $6 \mathrm{TFF}$ has an even stronger effect in the static probit model. Due to the negative effect of MDU, the graphs for a target rate increase (dashed line) and decrease (solid line) switch roles compared to variables with a positive influence. We further note that the effects in these figures are marginal effects, i.e., holding constant the other variables. However, we have previously seen that predictors seems to come in complementary sets. Hence, the marginal effects of IP and CCI may seem modest, but considered in conjuction with their complements, the joint impact will be larger.

A third finding from Table 3 is that the estimation results for the thresholds $\alpha_{1}$ and $\alpha_{2}$ also reveal a difference between the static and dynamic models. In the latter model, the posterior means of the thresholds are almost twice as large (in absolute value), substantially expanding the no-change region for the latent target rate. However, at least to some extent this is an implication of the difference in model specification. In the dynamic model, $r_{t}^{*}$ will be more variable in an absolute sense because the unconditional variance of the error $u_{t}$ is equal to $1 /\left(1-\varphi^{2}\right)>1$. To check whether the FOMC decides 'symmetrically' with regard to upward or downward target rate adjustments, we compute $\operatorname{Pr}\left[\alpha_{1}+\alpha_{2}>0 \mid \mathbf{y}\right]$. In case of symmetric behavior $\alpha_{1}+\alpha_{2}$ would equally likely be positive or negative. In the dynamic probit model this probability is 0.60 and the static model has a value of even 0.85 . This result supports our initial conjecture that the FOMC is more reluctant to increase the target rate than to decrease it (as $r_{t}^{*}$ must take more extreme values in order to trigger an increase of the target rate). 


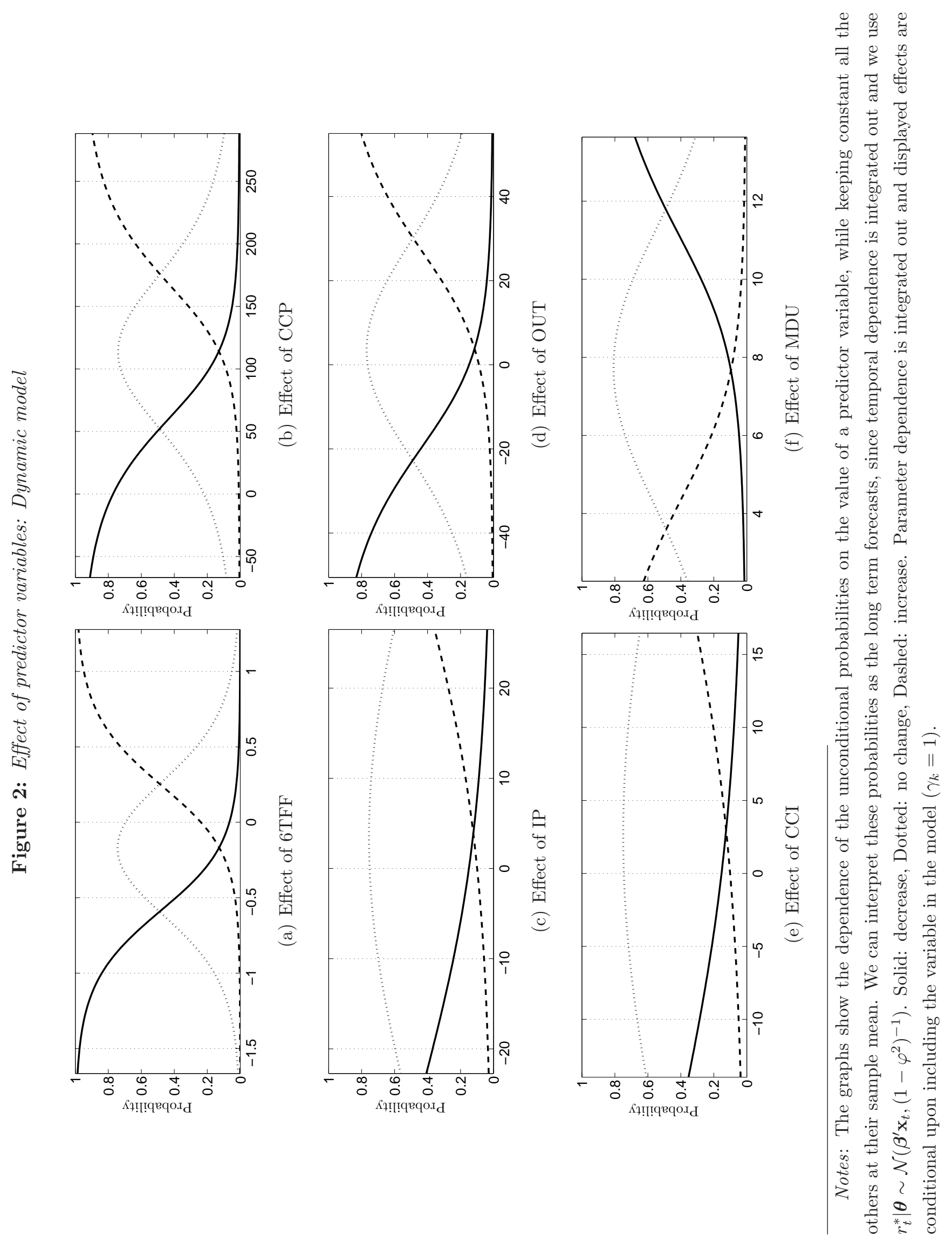



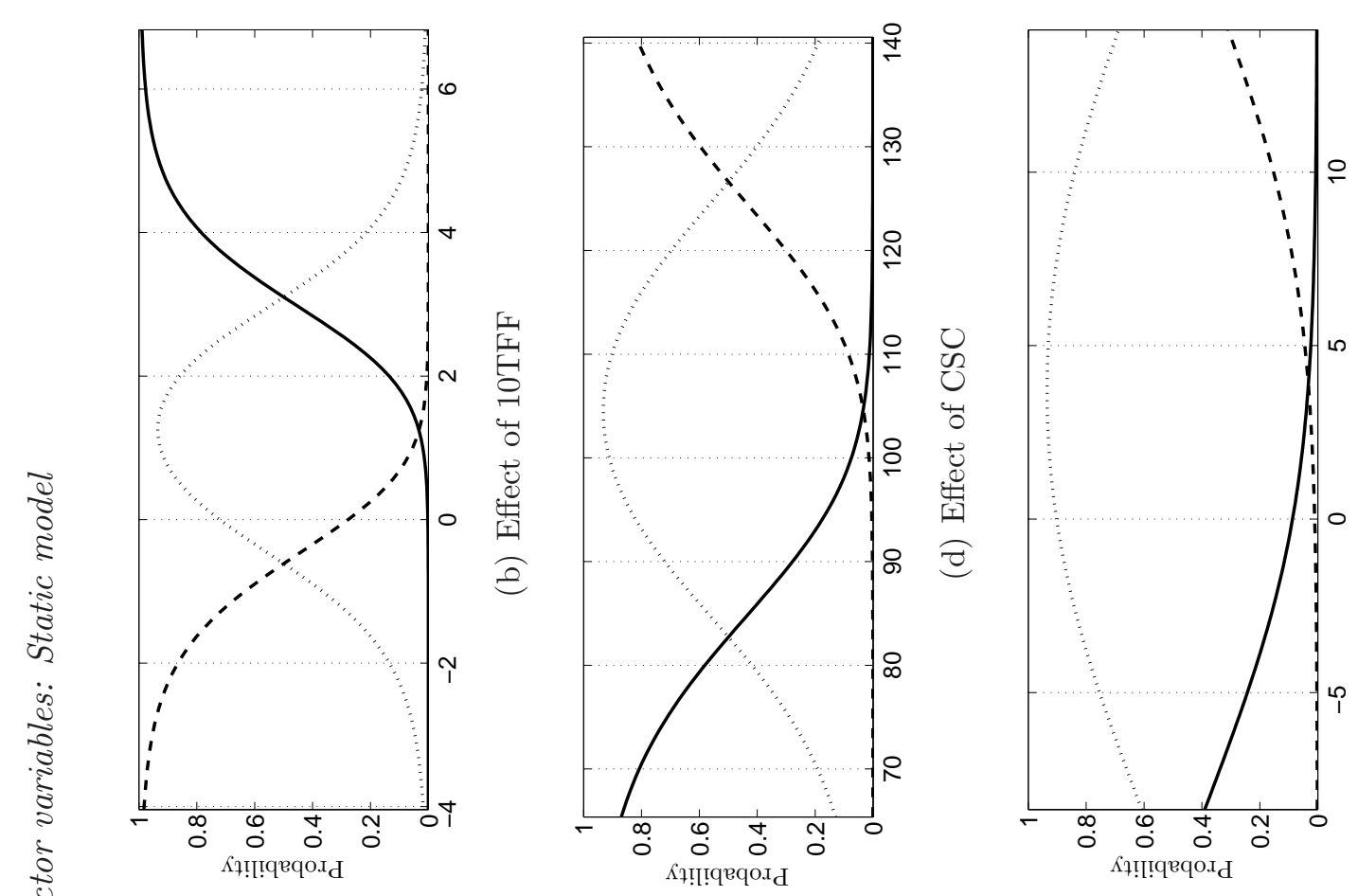

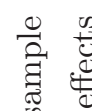

:

氠

娄

ज्ञे
0
0

章总

泀苛

工 $\begin{array}{ll}0 \\ 0 \\ 0\end{array}$

岁司

苞苟

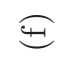

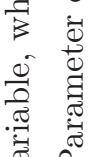

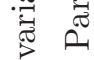

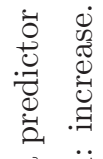

त

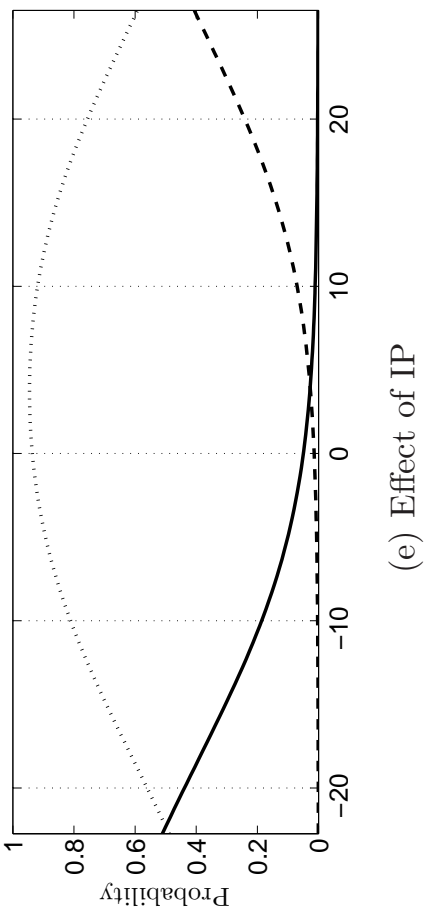

คิ

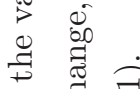

귱

焉

ही

岁跑.

苞

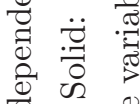

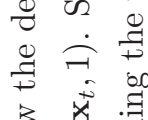

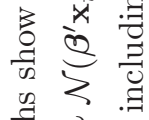

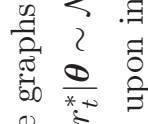

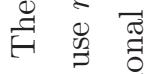

崩范苛 
Finally, Figure 4 shows the posterior time-path of the latent target rate $r_{t}^{*}$ and the thresholds that determine what decision will be taken. The latent target leads the announced target, with a maximum sample correlation (0.70) for a twelve-month lead time. This point is nicely illustrated by focusing on the period following the millennium change. Before the target was lowered in 2001 we observe that the latent rate had already been decreasing for several months. A similar pattern appears for the upward target rate adjustments from mid-2004 onwards. This leading characteristic is in line with previous research, see Hu and Phillips (2004). This graph also shows that large changes in the target rate $\left(\left|\Delta r_{t}\right|>.25\right)$ coincide with relatively large (absolute) values of $r_{t}^{*}$. For example, the rapid and pronounced decline in the latent rate during the second half of 2007 resulted in downward target changes of 0.50 and even 1.25 at single meetings. We note that this leading property of the latent target rate is quite promising for the ability of the dynamic probit model to actually predict future FOMC target rate decisions, which is the topic we turn to next.

Figure 4: Federal funds target rate, latent target rate and thresholds

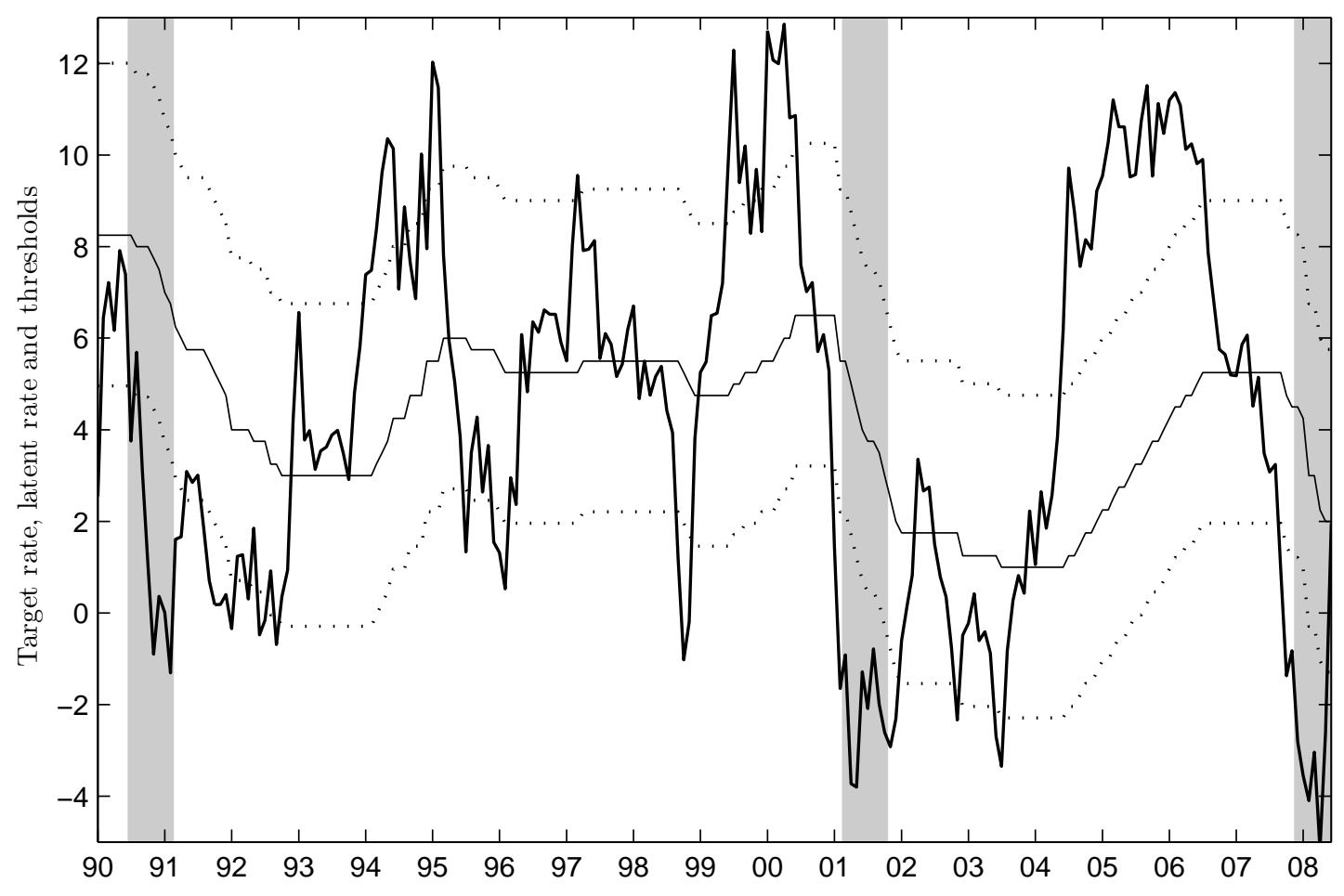




\section{$4.2 \quad$ Forecasting}

We examine the predictive ability of our dynamic ordered probit model in two ways. First, we compute the smoothed one-step ahead probabilities of a decrease, nochange or increase of the target rate for each month in the full sample period January 1990 - June 2008. Specifically, we compute the sample mean of $\operatorname{Pr}\left[y_{t}=j \mid \boldsymbol{\theta}, r_{t-1}^{*}\right]=$ $\Phi\left(\alpha_{j}+r_{t-1}-\mu_{t}\right)-\Phi\left(\alpha_{j-1}+r_{t-1}-\mu_{t}\right), \quad(j=1,2,3)$, over simulated values from the full-sample posterior $p\left(\boldsymbol{\theta}, \mathbf{r}^{*} \mid \mathbf{y}\right)$. We use that $\mu_{t}=\boldsymbol{\beta}^{\prime} \mathbf{x}_{t}+\varphi\left(r_{t-1}^{*}-\boldsymbol{\beta}^{\prime} \mathbf{x}_{t-1}\right)$ is the conditional mean of $r_{t}^{*}$. Figure 5 displays the results, with the shaded bars representing the actual FOMC decisions. The dynamic probit model fits very well, achieving a hitrate of 90 percent. The hitrate is defined as the percentage of correctly predicted target rate decisions, where the forecast is taken to be the decision that has the highest probability. If we consider the static probit model, the in-sample hitrate deteriorates with 16 percentage points to 74 percent. The dynamic probit probabilities show pronounced behavior, assigning most of the weight to a single decision. The static probit model generally gives more moderate probability forecasts for target rate increases and decreases and assigns more weight to the no-change decision. $^{13}$

Second, we employ our real-time Bayesian forecasting procedure and obtain genuine out-of-sample posterior predictive distributions for the period January 2001 June 2008. We depict the one-step ahead probabilities in Figure 6. We first focus on the target increases in the upper panel of Figure 6. The dynamic probit model succeeds in predicting the cluster of target rate increases from mid-2004 till mid-2006 rather well. Already at the start of 2004 the probability of a target rate hike gradually increases. In June, when the FOMC decided to increase the target rate for the first time, it was up to 0.5. For all other target rate increases in this period the probability forecast is in the range $[0.6 ; 0.9]$. For the first meeting after this period of consecutive target rate increases, in August 2006, the model predicts that a rise and no change would realize with probabilities 0.7 and 0.3 , respectively (see central panel of Figure 6). Hence, the probability of a target rate increase was already declining considerably.

Next, we turn attention to the target rate decreases in the lower panel of Figure 6. The probability forecasts for the beginning of the cluster in 2001 are not

\footnotetext{
${ }^{13}$ We do not display graphs of the smoothed probabilities for the static ordered probit model, but these are available upon request.
} 
very accurate. This holds especially for the decrease of June 2001 with a probability forecast of only 10 percent. Possibly the posterior was not yet precise enough to generate accurate forecasts, with only 88 meetings available in our sample before 2001. In-sample these meetings are fitted quite well though, as seen in Figure 5. During the following period, running from 2002 till the end of 2003, the target rate remained fairly stable with two isolated FOMC decisions to lower the target. A prolonged period of consecutive decreases of the target rate starting again at the end of 2007. During the six months before, the associated probability forecast already starts to increase. Following the final decrease in May 2008 this probability quickly returns to zero. We further note that for the whole sample, in periods of an increasing target rate, the probability of a decrease is virtually zero, and vice versa.

For the static probit model we find less pronounced probability forecasts. Especially during the period of consecutive increases in 2004-2006, the no-change decision is given considerable probability. For the period July 2002 - December 2003 the static model considers target rate decreases more likely than the dynamic model. Also for the static probit model, we find a substantial and persistent rise in the probabilities of a target increase or decrease already a few months before a period of such target rate changes actually starts.

We report out-of-sample hitrates and predictive likelihoods in Table 6. The dynamic probit model forecasts 82 percent of the meetings correctly. The static probit model achieves an out-of-sample hitrate of 77 percent. We conduct a formal evaluation of the models by computing the predictive likelihood $p\left(\mathbf{y}^{\tau+1, T} \mid \mathbf{y}^{1, \tau}\right)$ in the observed sample; $\tau+1$ is January 2001. The dynamic model takes a (log) value of -25.9 , compared to -40.0 for the static model, which shows that the dynamic variant finds far more support in the data.

To give some insight into the mechanics of the model averaging approach, we also estimate the models with a fixed set of regressors. For both the dynamic and static ordered probits we choose the posterior most likely predictor combination from Table 5. Note that it is $\boldsymbol{\beta}^{\prime} \mathbf{x}_{t}$ that determines the location of the normal distribution of $r_{t}^{*}$ in (3) and, therefore, the probabilities of the three possible decisions as determined by (2). Hence, if we allow for uncertainty in this part by allowing for uncertainty in the regressors that are included, these probabilities will on average be more spread compared to a case with fixed regressors. Although we cannot formally compare the predictive performance of the fixed regressor specification with the model averaging approach (as this is a situation with nested models), we find its 
Table 6: Hitrates and predictive likelihoods

\begin{tabular}{lllcc}
\hline \hline Model & Data & In-sample hitrate & Out-of-sample hitrate & Pred. likel. \\
\hline \multirow{2}{*}{$\begin{array}{l}\text { Dynamic } \\
\text { Static }\end{array}$} & Real time & $89.8(141 / 157)$ & $82.3(51 / 62)$ & -25.9 \\
Dynamic & Latest available & $88.5(139 / 157)$ & $77.4(48 / 62)$ & -40.0 \\
Static & Latest available & $77.7(122 / 157)$ & $70.6(50 / 62)$ & -26.7 \\
& & & & -33.6 \\
Benchmark 1: Time-invariant & $54.8(86 / 157)$ & $43.6(27 / 62)$ & -70.0 \\
Benchmark 2: Pure AR(1) & $81.5(128 / 157)$ & $85.5(53 / 62)$ & -33.2 \\
\hline \hline
\end{tabular}

Note: Hitrates are equal to the percentage of correctly predicted target rate decisions, when the forecast of the decision is given by $\hat{y}_{t}=\arg \max \left\{\operatorname{Pr}\left[y_{t}=j\right]: j=1,2,3\right\}$. The benchmarks are (1) a model with time-invariant probabilities $\boldsymbol{\pi}=\left(\pi_{1}, \pi_{2}, \pi_{3}\right)^{\prime}$ (effectively $\boldsymbol{\beta}=\mathbf{0}_{K}$ and $\varphi=0$ ) such that the dependent variables are temporally independent and $(2)$ a pure $\operatorname{AR}(1)$ model $\left(\boldsymbol{\beta}=\mathbf{0}_{K}\right)$ for the latent target rate $r_{t}^{*}$.

predictive likelihood to be higher. We also should stress that we do not choose an arbitrary model with fixed regressors, but the posterior most likely one. The advantage of model averaging becomes visible during the isolated target decreases in November 2002 and June 2003 though [Figure 6(c)]. These decisions get more weight compared to the fixed predictor specification. If we would like to have a more precise forecast distribution (i.e., more pronounced probabilities) in the future, we could use our posterior results as prior input in a new model. For example, the model space may be limited to the $J$ most likely predictor combinations. However, this more dogmatic prior comes with a cost as probabilities of less likely realizations (the ones in November 2002 and June 2003, for example) may be underestimated.

Finally we examine the difference between using revised data and data available in real time. Visual inspection of the probability plots of the dynamic model reveals no significant differences. In Table 6 we see that the predictive likelihood using real-time data is slightly larger compared to the latest-available data case. We can see an improvement in the in-sample fit of the static model if we switch from realtime to revised data. We find such an improvement in terms of the out-of-sample performance too. The predictive likelihood increases from -40.0 to -33.6 if we use latest-available instead of real-time data. 


\subsection{Robustness checks}

We assess the robustness of our empirical results in two respects. First, we examine the effect of the choice of prior on the predictor inclusion probability $\pi$. Second, we check the effects of changing the 'averaging' period $m$ used for constructing our predictor variables.

If we replace the uninformative Beta prior with a $\mathcal{B} e(3,1)$ (prior mean 0.75 but relatively large variance) or a $\mathcal{B} e(10,10)$ (prior mass relatively tightly concentrated around 0.5 ), the posterior mean of $\pi$ increases from 0.15 to 0.25 and 0.30 , respectively. The type of posterior most likely variables does not change though. These larger models also do not contribute to better forecasting results. Our findings support the idea that we should be careful with interpreting the posterior inclusion probabilities. These can easily be inflated via the prior without enhancing the fit of the model; the prior simply forces us to include irrelevant predictors more frequently.

The choice of the averaging period $m$ does have consequences. If we set it equal to three months $(m=3)$, similar predictors as for $m=1$ are selected. However, the forecasting performance deteriorates, mainly because the reversal decisions become harder to forecast. The smoothing of the predictors makes the probabilities to react more sluggish. An even more extreme choice of nine months has the result that hardly any explanatory variables provide useful information. A pure AR(1) process for $r_{t}^{*}$ is then the posterior most likely model. As a result the probabilities become very smooth with always considerable mass assigned to the no-change class. Obviously, since in this model hardly any exogenous information is selected, changing patterns are difficult to predict and we simply extrapolate the past. However, the predictive likelihood of a pure $\operatorname{AR}(1)$ for the latent target rate takes a value of -33.2 which is still quite an improvement over a benchmark model with time-invariant probabilities and a static ordered probit model, see Table 6 . We explain this by the strong dynamics in $r_{t}^{*}$ : once $y_{t}$ is in a particular class, outcomes of future meetings in the short run will likely be of the same type.

\section{Conclusions}

We model the discrete changes in the Federal funds target rate during the period January 1990 - June 2008. We focus on the direction of change as decided by the FOMC during their meetings held approximately eight times per year, using 
ordered probit models. The key modeling issue that we address concerns the question which indicators can help predict the FOMC decisions. For this reason we use an endogenous variable selection procedure. We consider a set of 33 potential predictors, including macroeconomic and financial series and forward-looking variables obtained from the Survey of Professional Forecasters.

Our empirical results show strong evidence for persistence in the target rate decisions, above the persistence caused by the (strongly) autocorrelated covariates. Endogenously selected explanatory variables are in line with previous literature. Most predictive ability is found for, first, economic activity measures like industrial production, the output gap and the coincident index, and, second, term structure variables like interest rate spreads. In addition we find that SPF-based forecasts for the three-month T-bill rate and different survey-based variables that measure current and future consumer confidence contain predictive content for the FOMC decisions. The estimation results furthermore suggest a kind of asymmetry in FOMC decision-making, in the sense that the FOMC seems more reluctant to increase than to decrease the target rate. Perhaps an erroneous slow-down could be considered more harmful than a bit of overstimulation.

Another contribution of this paper is that we propose a (Bayesian) forecasting scheme on a real-time basis to construct out-of-sample probability forecasts, efficiently using all information available at the time of generating the forecasts. For this purpose, we construct a real-time data set and update the posterior parameter beliefs with importance sampling each time a new observation comes in. The Bayesian approach makes sure that we can appropriately deal with parameter and model uncertainty to end up with a parameter- and model-free forecast.

Meetings in the period January 2001 - June 2008 are predicted well; 82 percent of the outcomes during the out-of-sample period 2001-2008 are correctly predicted. In-sample we even achieve a hitrate of 90 percent. Changes in the economy and subsequently in the outcome of FOMC meetings are quickly incorporated in the forecasts. For example, before the FOMC started increasing the target rate in mid2004, its probability forecast was already rising for four months. The dynamic ordered probit model improves forecasting performance substantially compared to a static model, which achieves hitrates of 77 and 74 percent. 


\section{References}

Albert, J. H. and Chib, S. (1993). Bayesian analysis for binary and polychotomous response data, Journal of the American Statistical Association 88: 901-921.

Andersen, T. G., Bollerslev, T., Diebold, F. X. and Vega, C. (2007). Real-time price discovery in stock, bond and foreign exchange markets, Journal of International Economics 73: 251-277.

Ang, A., Bekaert, G. and Wei, M. (2007). Do macro variables, asset markets, or surveys forecast inflation better?, Journal of Monetary Economics 54: 1163-1212.

Bernanke, B. S. and Blinder, A. S. (1992). The federal funds rate and the channels of monetary transmission, American Economic Review 82: 901-921.

Bernanke, B. S. and Kuttner, K. N. (2005). What explains the stock market's reaction to Federal Reserve policy?, Journal of Finance 60: 1221-1257.

Campbell, S. D. and Diebold, F. X. (2009). Stock returns and expected business conditions: Half a century of direct evidence, Journal of Business and Economic Statistics 27: 266-278.

Chib, S. and Greenberg, E. (1994). Bayes inference in regression models with $\operatorname{ARMA}(\mathrm{p}, \mathrm{q})$ errors, Journal of Econometrics 64: 183-206.

Chulia-Soler, H., Martens, M. and van Dijk, D. (2010). Asymmetric effects of federal funds target rate changes on S\&P100 stock returns, volatilities and correlations, Journal of Banking and Finance 34: 834-839.

Croushore, D. (1993). Introducing: the survey of professional forecasters, Federal Reserve Bank of Philadelphia Business Review pp. 3-15.

Croushore, D. (2006). Forecasting with real-time macroeconomic data, in G. Elliott, C. W. J. Granger and A. Timmermann (eds), Handbook of Economic Forecasting, Volume 1, North-Holland, Amsterdam, pp. 961-982.

Croushore, D. and Stark, T. (2001). A real-time data set for macroeconomists, Journal of Econometrics 105: 111-130. 
Diebold, F. X. and Rudebusch, G. D. (1991). Forecasting output with the composite leading index: An ex ante analysis, Journal of the American Statistical Association 86: $603-610$.

Doppelhofer, G. and Weeks, M. (2009a). Jointness of growth determinants, Journal of Applied Econometrics 24: 209-244.

Doppelhofer, G. and Weeks, M. (2009b). Jointness of growth determinants: Reply to comments by Rodney Strachan, Eduardo Ley and Mark F.J. Steel, Journal of Applied Econometrics 24: 252-256.

Dueker, M. (1999). Measuring monetary policy inertia in target Fed funds rate changes, Federal Reserve Bank of St. Louis Review pp. 3-9.

Dueker, M. J. and Rasche, R. H. (2004). Discrete policy changes and empirical models of the Federal funds rate, Federal Reserve Bank of St. Louis Review pp. 6172.

Ehrmann, M. and Fratzscher, M. (2007). Transparency, disclosure and the Federal Reserve, International Journal of Central Banking 3: 179-225.

Estrella, A. and Mishkin, F. S. (1997). The predictive power of the term structure of interest rates in Europe and the United States: Implications for the European Central Bank, European Economic Review 41: 1375-1401.

Faust, J., Rogers, J. H., Wang, S.-Y. B. and Wright, J. H. (2007). The high-frequency response of exchange rates and interest rates to macroeconomic announcements, Journal of Monetary Economics 54: 1051-1068.

Federal Reserve Board of Governors (2005). The Federal Reserve System - Purposes and Functions.

George, E. I. and McCulloch, R. E. (1993). Variable selection via Gibbs sampling, Journal of the American Statistical Association 88: 901-921.

Geweke, J. (2005). Contemporary Bayesian Econometrics and Statistics, John Wiley \& Sons, Hoboken, NJ.

Grammig, J. and Kehrle, K. (2008). A new marked point process model for the federal funds rate target - Methodology and forecast evaluation, Journal of Economic Dynamics \& Control 32: 2370-2396. 
Hamilton, J. D. and Jordà, O. (2002). A model of the Federal funds rate target, Journal of Political Economy 110: 1135-1167.

Hayo, B. and Neuenkirch, M. (2010). Do Federal Reserve communications help predict federal funds target rate decisions?, Journal of Macroeconomics 32: 10141024.

Hu, L. and Phillips, P. C. B. (2004). Dynamics of the Federal funds target rate: a nonstationary discrete choice approach, Journal of Applied Econometrics 19: 851867.

Kauppi, H. (forthcoming). Predicting the direction of the Fed's target rate, Journal of Forecasting .

Kim, H., Jackson, J. and Saba, R. (2009). Forecasting the FOMC's interest rate setting behavior: A further analysis, Journal of Forecasting 28: 145-165.

Kuo, L. and Mallick, B. (1998). Variable selection for regression models, The Indian Journal of Statistics 60: 901-921.

Liu, J. S. and Sabatti, C. (2000). Generalised Gibbs sampler and multigrid Monte Carlo for Bayesian computation, Biometrika 87: 901-921.

Mishkin, F. S. (1990a). The information in the longer-maturity term structure about future inflation, Quarterly Journal of Economics 55: 815-828.

Mishkin, F. S. (1990b). What does the term structure tell us about future inflation?, Journal of Monetary Economics 25: 77-95.

Monokroussos, G. (2011). Dynamic limited dependent variable modeling and U.S. monetary policy, Journal of Money, Credit and Banking 43: 519-534.

Piazzesi, M. (2005). Bond yields and the Federal Reserve, Journal of Political Economy 113: 311-344.

Poole, W. (2005). How predictable is Fed policy?, Federal Reserve Bank of St.Louis Review Nov/Dec: 659-668.

Robert, C. P. and Casella, G. (2004). Monte Carlo Statistical Methods, 2nd edn, Springer-Verlag, New York, NY. 
Rudebusch, G. D. (2001). Is the Fed too timid? Monetary policy in an uncertain world, Review of Economics and Statistics 81: 203-217.

Rudebusch, G. D. (2002). Term structure evidence on interest rate smoothing and monetary policy inertia, Journal of Monetary Economics 49: 1161-1187.

Stark, T. and Croushore, D. (2002). Forecasting with a real-time data set for macroeconomists, Journal of Macroeconomics 24: 507531.

Stock, J. H. and Watson, M. W. (2003). Forecasting output and inflation: The role of asset prices, Journal of Economic Literature 41: 788-829.

Tanner, M. A. and Wong, W. H. (1987). The calculation of posterior distibutions by data augmentation, Journal of the American Statistical Association 82: 528-550.

Taylor, J. B. (1993). Discretion versus policy rules in practice, Carnegie-Rochester Conference Series on Public Policy 39: 195-214.

Tierney, L. (1994). Markov chains for exploring posterior distributions, Annals of Statistics 22: 1701-1762.

Vanderhart, P. G. (2000). The Federal Reserve's reaction function under Greenspan: An ordinal probit analysis, Journal of Macroeconomics 22: 631-644. 


\section{Appendix A Posterior simulation}

In this appendix we provide details of the main components for the Bayesian analysis of our dynamic ordered probit model. We specify the functional forms of the prior and the complete data likelihood in Section A.1. In Section A.2 we discuss the details of the Markov chain Monte Carlo simulation scheme.

\section{A.1 Prior distribution and complete data likelihood}

The joint prior density for the parameters follows from the individual priors specified in Section 2.2:

$$
\begin{aligned}
p(\boldsymbol{\theta}) \propto & \exp \left\{-\frac{1}{2}(\boldsymbol{\psi}-\mathbf{a})^{\prime} \mathbf{A}^{-1}(\boldsymbol{\psi}-\mathbf{a})\right\} \exp \left\{-\frac{1}{2 B}(\varphi-b)^{2}\right\} \times \mathbb{I}_{\{\varphi \in S\}} \\
& \times \pi^{c_{1}+N(\boldsymbol{\gamma})-1}(1-\pi)^{c_{2}+K-N(\boldsymbol{\gamma})-1} \times \mathbb{I}_{\left\{\alpha_{1}<\alpha_{2}\right\}},
\end{aligned}
$$

and $N(\gamma)=\sum_{k=1}^{K} \gamma_{k}$ is the number of included covariates.

To derive the complete data likelihood of our model (1)-(4) we collect the latent target rates $r_{t}^{*}$ in $\mathbf{r}^{*}=\left(r_{1}^{*}, \ldots, r_{T}^{*}\right)^{\prime}$ and the observed target rate decisions $y_{t}$ in the vector $\mathbf{y}=\left(y_{1}, \ldots, y_{T}\right)^{\prime}$. The complete data likelihood is decomposed as $p\left(\mathbf{y}, \mathbf{r}^{*} \mid \boldsymbol{\theta}\right)=$ $p\left(\mathbf{y} \mid \mathbf{r}^{*}, \boldsymbol{\theta}\right) p\left(\mathbf{r}^{*} \mid \boldsymbol{\theta}\right)$ and the first pdf on the left equals

$$
p\left(\mathbf{y} \mid \mathbf{r}^{*}, \boldsymbol{\theta}\right)=\prod_{t=1}^{T} p\left(y_{t} \mid r_{t}^{*}, \boldsymbol{\theta}\right)=\prod_{t=1}^{T} \mathbb{I}_{\left\{r_{t}^{*}-r_{t-1} \in\left(\alpha_{y_{t}-1}, \alpha_{y_{t}}\right]\right\}}
$$

in which the latter equality follows from the mapping rule in (2); conditional on $r_{t}^{*}$, $y_{t}$ is degenerate. To derive the other pdf, $p\left(\mathbf{r}^{*} \mid \boldsymbol{\theta}\right)$, we use the first-order Markov property of $\left\{r_{t}^{*}\right\}$ by combining (3) and (4), which results in

$$
p\left(\mathbf{r}^{*} \mid \boldsymbol{\theta}\right)=p\left(r_{1}^{*} \mid \boldsymbol{\theta}\right) \prod_{t=2}^{T} p\left(r_{t}^{*} \mid r_{t-1}^{*}, \boldsymbol{\theta}\right)
$$

If we use (3) and (4), then the individual conditional densities in (A.3) are Gaussian with means

$$
\mu_{t}=\boldsymbol{\beta}^{\prime} \mathbf{x}_{t}+\varphi\left(r_{t-1}^{*}-\boldsymbol{\beta}^{\prime} \mathbf{x}_{t-1}\right)
$$

and unit variances, for $t=2, \ldots, T$. We model the first latent variable $r_{1}^{*}$ using its marginal distribution (given $\mathbf{x}_{1}$ ) and hence

$$
r_{1}^{*}=\boldsymbol{\beta}^{\prime} \mathbf{x}_{1}+u_{1}, \quad \text { with } \quad u_{1} \sim \mathcal{N}\left(0, \frac{1}{1-\varphi^{2}}\right)
$$


Both (A.2) and (A.3)-(A.5) reveal the complete data likelihood:

$$
\begin{aligned}
p\left(\mathbf{y}, \mathbf{r}^{*} \mid \boldsymbol{\theta}\right) \propto & \prod_{t=1}^{T} \mathbb{I}_{\left\{r_{t}^{*}-r_{t-1} \in\left(\alpha_{y_{t}-1}, \alpha_{y_{t}}\right]\right\}} \times\left(1-\varphi^{2}\right)^{\frac{1}{2}} \exp \left\{-\frac{1-\varphi^{2}}{2}\left(r_{1}^{*}-\boldsymbol{\beta}^{\prime} \mathbf{x}_{1}\right)^{2}\right\} \\
& \times \exp \left\{-\frac{1}{2} \sum_{t=2}^{T}\left(r_{t}^{*}-\boldsymbol{\beta}^{\prime} \mathbf{x}_{t}-\varphi\left(r_{t-1}^{*}-\boldsymbol{\beta}^{\prime} \mathbf{x}_{t-1}\right)\right)^{2}\right\},
\end{aligned}
$$

in which the integrating constant does not depend on parameters $\boldsymbol{\theta}$.

\section{A.2 Posterior simulation steps}

We set up an MCMC sampler to simulate from the posterior $p\left(\boldsymbol{\theta}, \mathbf{r}^{*} \mid \mathbf{y}\right)$ which is proportional to the product of (A.1) and (A.6). We simulate the parameters and the latent variables from their full conditional posterior distributions. The simulation scheme is provided in Section 2.3. The first three steps of the sampler are standard. Steps 4 and 5-6 are discussed in detail.

\section{Step 4: Sampling latent data}

The latent target behaves like $r_{t}^{*}-\boldsymbol{\beta}^{\prime} \mathbf{x}_{t}=\varphi\left(r_{t-1}^{*}-\boldsymbol{\beta}^{\prime} \mathbf{x}_{t-1}\right)+\varepsilon_{t}$ with $\varepsilon_{i} \stackrel{i . i . d .}{\sim} \mathcal{N}(0,1)$. This temporal dependence in combination with the truncation (caused by conditioning on the observed categories) makes the simulation of all latent variables in one block impossible. Instead, we sample them individually from their full conditional posteriors, see Albert and Chib (1993) and Geweke (2005).

We start with the initial $r_{1}^{*}$. Since the process for $r_{t}^{*}$ is first-order Markov all relevant information in the series for $r_{1}^{*}$ is captured by $r_{2}^{*}$. We use the following two equations to derive the full conditional posterior of $r_{1}^{*}$ :

$$
\begin{aligned}
r_{1}^{*} & =\boldsymbol{\beta}^{\prime} \mathbf{x}_{1}+u_{1}, \\
r_{2}^{*} & =\boldsymbol{\beta}^{\prime} \mathbf{x}_{2}+\varphi\left(r_{1}^{*}-\boldsymbol{\beta}^{\prime} \mathbf{x}_{1}\right)+\varepsilon_{2} .
\end{aligned}
$$

This system is rewritten as

$$
\begin{aligned}
\sqrt{1-\varphi^{2}} \boldsymbol{\beta}^{\prime} \mathbf{x}_{1} & =\sqrt{1-\varphi^{2}} r_{1}^{*}+\varepsilon_{1}, \\
r_{2}^{*}-\boldsymbol{\beta}^{\prime} \mathbf{x}_{2}+\varphi \boldsymbol{\beta}^{\prime} \mathbf{x}_{1} & =\varphi r_{1}^{*}+\varepsilon_{2},
\end{aligned}
$$

with $\varepsilon_{i} \stackrel{i . i . d .}{\sim} \mathcal{N}(0,1),(i=1,2)$. Hence, we have a normal linear regression framework with dependent and independent variables

$$
\mathbf{y}_{1}^{*}=\left[\begin{array}{c}
\sqrt{1-\varphi^{2}} \boldsymbol{\beta}^{\prime} \mathbf{x}_{1}, \\
r_{2}^{*}+\boldsymbol{\beta}^{\prime}\left(\varphi \mathbf{x}_{1}-\mathbf{x}_{2}\right)
\end{array}\right], \quad \mathbf{X}_{1}^{*}=\left[\begin{array}{c}
\sqrt{1-\varphi^{2}} \\
\varphi
\end{array}\right],
$$


respectively. This result shows that $r_{1}^{*}$ should be sampled from a normal distribution with mean $\left(\mathbf{X}_{1}^{*^{\prime}} \mathbf{X}_{1}^{*}\right)^{-1} \mathbf{X}_{1}^{*^{\prime}} \mathbf{y}_{1}^{*}$ and variance $\left(\mathbf{X}_{1}^{*^{\prime}} \mathbf{X}_{1}^{*}\right)^{-1}=1$, truncated to the interval $\left(r_{0}+\alpha_{y_{1}-1}, r_{0}+\alpha_{y_{1}}\right]$.

For $r_{t}^{*},(t=2, \ldots, T-1)$, we derive the full conditional posteriors in a similar fashion. We collect the $r_{t}^{*}$-terms from the joint posterior and get the system:

$$
\begin{aligned}
\boldsymbol{\beta}^{\prime} \mathbf{x}_{t}+\varphi\left(r_{t-1}^{*}-\boldsymbol{\beta}^{\prime} \mathbf{x}_{t-1}\right) & =r_{t}^{*}+\varepsilon_{t}, \\
r_{t+1}^{*}-\boldsymbol{\beta}^{\prime} \mathbf{x}_{t+1}+\varphi \boldsymbol{\beta}^{\prime} \mathbf{x}_{t} & =\varphi r_{t}^{*}+\varepsilon_{t+1} .
\end{aligned}
$$

If we define the auxiliary variables

$$
\mathbf{y}_{t}^{*}=\left[\begin{array}{c}
\varphi r_{t-1}^{*}+\boldsymbol{\beta}^{\prime}\left(\mathbf{x}_{t}-\varphi \mathbf{x}_{t-1}\right) \\
r_{t+1}^{*}+\boldsymbol{\beta}^{\prime}\left(\varphi \mathbf{x}_{t}-\mathbf{x}_{t+1}\right)
\end{array}\right], \quad \mathbf{X}_{t}^{*}=\left[\begin{array}{c}
1 \\
\varphi
\end{array}\right],
$$

then $r_{t}^{*}$ is sampled from a normal distribution with mean $\left(\mathbf{X}_{t}^{*^{\prime}} \mathbf{X}_{t}^{*}\right)^{-1} \mathbf{X}_{t}^{*^{\prime}} \mathbf{y}_{t}^{*}$ and variance $\left(\mathbf{X}_{t}^{*^{\prime}} \mathbf{X}_{t}^{*}\right)^{-1}=1 /\left(1+\varphi^{2}\right)$, truncated to the interval $\left(r_{t-1}+\alpha_{y_{t}-1}, r_{t-1}+\alpha_{y_{t}}\right]$.

At the end of the sample period there is only one data point that contains information for $r_{T}^{*}$ :

$$
r_{T}^{*}=\boldsymbol{\beta}^{\prime} \mathbf{x}_{T}+\varphi\left(r_{T-1}^{*}-\boldsymbol{\beta}^{\prime} \mathbf{x}_{T-1}\right)+\varepsilon_{T} .
$$

We sample this final latent variable from a normal with mean $\boldsymbol{\beta}^{\prime} \mathbf{x}_{T}+\varphi\left(r_{T-1}^{*}-\right.$ $\left.\boldsymbol{\beta}^{\prime} \mathbf{x}_{T-1}\right)$, unit variance and truncated to the interval $\left(r_{T-1}+\alpha_{y_{T}-1}, r_{T-1}+\alpha_{y_{T}}\right]$.

\section{Steps 5-6: Variable selection}

In order to apply the Kuo and Mallick (1998) step we rewrite the system such that, conditional on $\left\{\mathbf{r}^{*}, \varphi\right\}$, a normal linear regression framework appears. We construct adjusted dependent, $v_{t}$, and independent, $\mathbf{w}_{t}$, variables by collecting terms involving $\boldsymbol{\beta}$ :

$$
\begin{aligned}
& v_{t} \equiv r_{t}^{*}-\varphi r_{t-1}^{*}=\boldsymbol{\beta}^{\prime}\left(\mathbf{x}_{t}-\varphi \mathbf{x}_{t-1}\right)+\varepsilon_{t}=\boldsymbol{\beta}^{\prime} \mathbf{w}_{t}+\varepsilon_{t}, \quad(t=2, \ldots, T), \\
& v_{1} \equiv \sqrt{1-\varphi^{2}} r_{1}^{*}=\sqrt{1-\varphi^{2}} \boldsymbol{\beta}^{\prime} \mathbf{x}_{1}+\varepsilon_{1}=\boldsymbol{\beta}^{\prime} \mathbf{w}_{1}+\varepsilon_{1}
\end{aligned}
$$

With this result we can perform the variable selection in the normal linear regression setup $\mathbf{v}=\mathbf{W} \boldsymbol{\beta}+\boldsymbol{\varepsilon}$ with dependent variables $\mathbf{v}=\left(v_{1}, \ldots, v_{T}\right)^{\prime}$ and explanatory variables $\mathbf{W}=\left(\mathbf{w}_{1}, \ldots, \mathbf{w}_{T}\right)^{\prime}$. First we sample the inclusion indicator $\gamma_{k}$ from its full conditional posterior for all $k$ (in random order), which is Bernoulli. Next we update the regression parameters $\boldsymbol{\psi}$ in one block (conditional on the new inclusion indicators), for which we draw from their joint multivariate normal distribution. 


\section{Appendix B Bayesian forecasting}

In this appendix we discuss the details of the implementation of the real-time Bayesian forecasting procedure. During this forecasting exercise we want to generate a series of one-step ahead predictive densities $p\left(y_{s} \mid \mathbf{y}^{1, s-1}\right)$ for $s=\tau+1, \ldots, T$, given by

$$
p\left(y_{s} \mid \mathbf{y}^{1, s-1}\right)=\int p\left(y_{s} \mid \boldsymbol{\theta}, \mathbf{r}^{* ; 1, s-1}, \mathbf{y}^{1, s-1}\right) p\left(\boldsymbol{\theta}, \mathbf{r}^{* ; 1, s-1} \mid \mathbf{y}^{1, s-1}\right) d \mathbf{r}^{* ; 1, s-1} d \boldsymbol{\theta} .
$$

As discussed in Section 2.3, we need a sample from $p\left(\boldsymbol{\theta}, \mathbf{r}^{* ; 1, s-1} \mid \mathbf{y}^{1, s-1}\right)$ to be able to evaluate these predictive densities. To avoid calculating posterior results for every time period $s$, we opt for importance sampling techniques (see, for example, Robert and Casella; 2004, for a discussion).

The construction of the predictive density of $y_{s}$ requires a sample from the posterior distribution with pdf $p\left(\boldsymbol{\theta}, \mathbf{r}^{* ; 1, s-1} \mid \mathbf{y}^{1, s-1}\right)$, which we decompose as

$$
p\left(\boldsymbol{\theta}, \mathbf{r}^{* ; 1, s-1} \mid \mathbf{y}^{1, s-1}\right)=p\left(r_{s-1}^{*} \mid \boldsymbol{\theta}, \mathbf{r}^{* ; 1, s-2}, \mathbf{y}^{1, s-1}\right) p\left(\boldsymbol{\theta}, \mathbf{r}^{* ; 1, s-2} \mid \mathbf{y}^{1, s-1}\right) .
$$

Given a sample from $p\left(\boldsymbol{\theta}, \mathbf{r}^{* ; 1, s-2} \mid \mathbf{y}^{1, s-1}\right)$, we draw $r_{s-1}^{*}$ from $p\left(r_{s-1}^{*} \mid \boldsymbol{\theta}, \mathbf{r}^{* ; 1, s-2}, \mathbf{y}^{1, s-1}\right)$. The latter simplifies to $p\left(r_{s-1}^{*} \mid \boldsymbol{\theta}, r_{s-2}^{*}, y_{s-1}\right)$ and is the pdf of a normal distribution with mean $\mu_{s-1}$ defined in (A.4), unit variance and truncated to the region $\left(r_{s-2}+\right.$ $\left.\alpha_{y_{s-1}-1}, r_{s-2}+\alpha_{y_{s-1}}\right]$.

To sample from $p\left(\boldsymbol{\theta}, \mathbf{r}^{* ; 1, s-2} \mid \mathbf{y}^{1, s-1}\right)$ we use importance sampling. As importance function we apply the posterior density using data up to and including period $s-2$, that is, $p\left(\boldsymbol{\theta}, \mathbf{r}^{* ; 1, s-2} \mid \mathbf{y}^{1, s-2}\right)$. We write

$$
p\left(\boldsymbol{\theta}, \mathbf{r}^{* ; 1, s-2} \mid \mathbf{y}^{1, s-1}\right)=\frac{p\left(y_{s-1} \mid \boldsymbol{\theta}, \mathbf{r}^{* ; 1, s-2}, \mathbf{y}^{1, s-2}\right) p\left(\boldsymbol{\theta}, \mathbf{r}^{* ; 1, s-2} \mid \mathbf{y}^{1, s-2}\right) p\left(\mathbf{y}^{1, s-2}\right)}{p\left(\mathbf{y}^{1, s-1}\right)}
$$

and hence the importance weights are given by

$$
w_{s}\left(\boldsymbol{\theta}, \mathbf{r}^{* ; 1, s-2}\right)=\frac{p\left(y_{s-1} \mid \boldsymbol{\theta}, \mathbf{r}^{* ; 1, s-2}, \mathbf{y}^{1, s-2}\right)}{p\left(y_{s-1} \mid \mathbf{y}^{1, s-2}\right)} .
$$

This whole setup is applied recursively and we start with a posterior sample from $p\left(\boldsymbol{\theta}, \mathbf{r}^{* ; 1, \tau} \mid \mathbf{y}^{1, \tau}\right)$. We compute the predictive density for $y_{\tau+1}$ using Monte Carlo integration. For the periods $s=\tau+2, \ldots, T$, we keep on simulating the latent variables $r_{s-1}^{*}$ and correct with importance weights according to the following scheme:

Step 1. Given a draw of $\left\{\boldsymbol{\theta}, \mathbf{r}^{* ; 1, s-2}\right\}$ simulate $r_{s-1}^{*}$ from $p\left(r_{s-1}^{*} \mid \boldsymbol{\theta}, \mathbf{r}^{* ; 1, s-2}, \mathbf{y}^{1, s-1}\right)$, which is $\mathcal{N}\left(\mu_{s-1}, 1\right)$ truncated to $\left(r_{s-2}+\alpha_{y_{s-1}-1}, r_{s-2}+\alpha_{y_{s-1}}\right]$ where $\mu_{s-1}$ is given in (A.4); 
Step 2. Update the importance weight $w_{s}\left(\boldsymbol{\theta}, \mathbf{r}^{* ; 1, s-2}\right)$ using

$$
w_{s}\left(\boldsymbol{\theta}, \mathbf{r}^{* ; 1, s-2}\right)=\frac{p\left(y_{s-1} \mid \boldsymbol{\theta}, \mathbf{r}^{* ; 1, s-2}, \mathbf{y}^{1, s-2}\right)}{p\left(y_{s-1} \mid \mathbf{y}^{1, s-2}\right)} w_{s-1}\left(\boldsymbol{\theta}, \mathbf{r}^{* ; 1, s-3}\right),
$$

with the initialization $w_{\tau+1}=1$;

Step 3. Evaluate

$$
\operatorname{Pr}\left[y_{s}=j \mid \boldsymbol{\theta}, r_{s-1}^{*}\right] \cdot w_{s}=\left[\Phi\left(\alpha_{j}+r_{s-1}-\mu_{s}\right)-\Phi\left(\alpha_{j-1}+r_{s-1}-\mu_{s}\right)\right] \cdot w_{s},
$$

in the simulated value of $\left\{\boldsymbol{\theta}, \mathbf{r}^{* ; 1, s-1}\right\}$ for all possible categories $(j=1,2,3)$, where $\mu_{s}$ is given in (A.4) and $\Phi(\cdot)$ is the standard normal cumulative distribution function;

Step 4. Repeat the previous three steps $M$ (= number of simulation runs) times and compute $p\left(y_{s} \mid \mathbf{y}^{1, s-1}\right)$ using the simulation sample average over the values in Step 3.

\section{Appendix C An alternative dependent variable}

The FOMC meets eight times per year, approximately every six weeks. For our ordered probit model, which is specified in calendar time at a monthly frequency, this implies that on average there are four observations each year for which there is no formal decision on the target rate. In the main analysis we treat those months as giving rise to missing observations for the dependent variable $y_{t}$ as defined in (1). In this appendix we briefly summarize the ordered probit results obtained with an alternative treatment of those months. Specifically, we set $y_{t}=2$ when no FOMC meeting takes place in month $t$, corresponding with the target rate remaining at the same level. This alternative definition may be motivated by noting that if there had been reason to change the target rate, the FOMC would have done so by means of an unscheduled meeting.

An immediate consequence of this alternative way of handling months without an FOMC meeting is that the latent target rate $r_{t}^{*}$ is forced to be such that the difference with $r_{t-1}$ remains within the interval $\left(\alpha_{1}, \alpha_{2}\right]$, due to the relation between $y_{t}$ and $r_{t}^{*}$ in (2). This leads to erratic behavior of $r_{t}^{*}$, especially during prolonged periods of consecutive FOMC decisions to increase or decrease the target rate. This can be seen from the posterior time-path of $r_{t}^{*}$ in Figure C.1. As an example, between 
June 2004 and June 2006, the FOMC decided to increase the target rate by 25 basis points at each of the 17 meetings that were held during this period. Nevertheless we observe that $r_{t}^{*}-r_{t-1}$ did not increase much beyond the threshold $\alpha_{2}$ during the months with FOMC meetings in this period. This results from the fact that $r_{t}^{*}-r_{t-1}$ necessarily falls below $\alpha_{2}$ during the months without a meeting.

The main implication of this behavior of $r_{t}^{*}$ is that by construction the ordered probit model cannot provide a strong signal for target rate increases or decreases. Again, during the period June 2004 - June 2006, the difference between the latent target rate and the prevailing actual target rate $r_{t-1}$ never becomes much larger than the threshold $\alpha_{2}$, such that the probability of no target rate change will always be quite substantial. This is confirmed by Figure C.2, which shows the smoothed in-sample probabilities for the three categories of target rate decisions based on estimating the dynamic probit model using the complete sample period. The probability of a target rate increase or decrease typically hardly ever exceeds 0.7.

This effect is felt even more strongly when the model is used for out-of-sample forecasting. Figure C.3 shows one-month ahead real-time out-of-sample probabilities from the dynamic probit model for the period January 2001 - June 2008. With few exceptions, the probability forecast for a target rate decrease or increase does not become larger than 0.5 .

Not surprisingly then, the forecast performance of the ordered probit models deteriorates considerably. Table C.1 shows the in-sample and out-of-sample hit rates obtained from the dynamic and static models with real time and latest available data. For all specifications, the hitrates are 10-20 percent lower than those obtained in the main analysis, as shown in Table 6 . 
Table C.1: Alternative $y_{t}$ : Hitrates and predictive likelihoods

\begin{tabular}{llccc}
\hline \hline Model & Data & In-sample hitrate & Out-of-sample hitrate & Pred. likel. \\
\hline \multirow{2}{*}{$\begin{array}{l}\text { Dynamic } \\
\text { Static }\end{array}$} & Real time & $77.9(173 / 222)$ & $67.8(61 / 90)$ & -61.4 \\
Dynamic & Latest available & $71.6(159 / 222)$ & $61.1(55 / 90)$ & -67.7 \\
Static & Latest available & $69.8(158 / 222)$ & $57.8(52 / 90)$ & -64.7 \\
& & & $64.4(58 / 90)$ & -60.1 \\
Benchmark 1: Time-invariant & $68.0(151 / 222)$ & $61.1(55 / 90)$ & -86.7 \\
Benchmark 2: Pure AR(1) & $69.8(155 / 222)$ & $64.4(58 / 90)$ & -63.2 \\
\end{tabular}

Note: The table shows the hitrates and predictive likelihoods for the models with the alternative $y_{t}$. Since we do not have missing observations in this setting, we have 222 observations in-sample and 90 FOMC decisions out-of-sample. Also note that we cannot compare these values with the ones in Table 6 . For further notes, see Table 6 . 

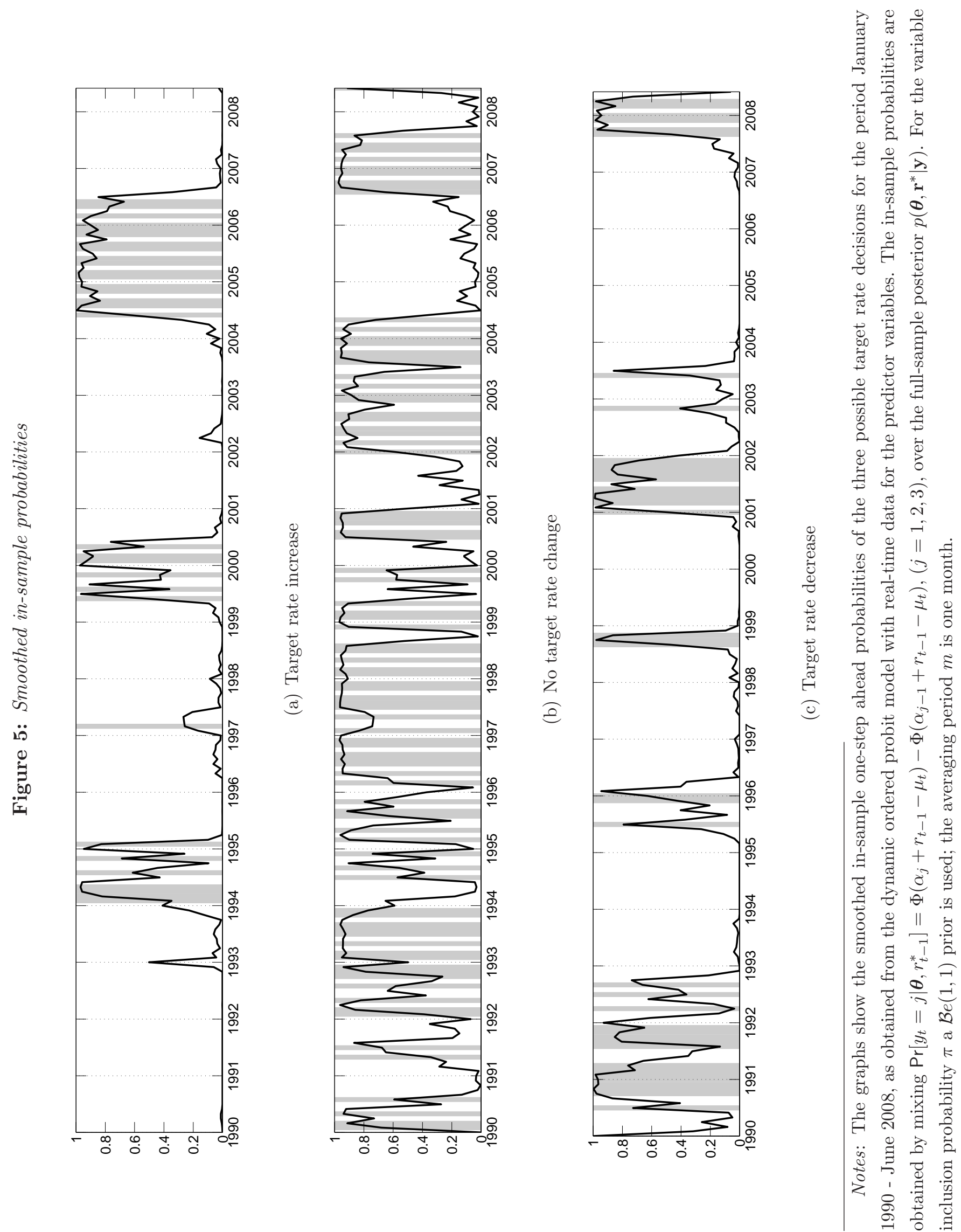

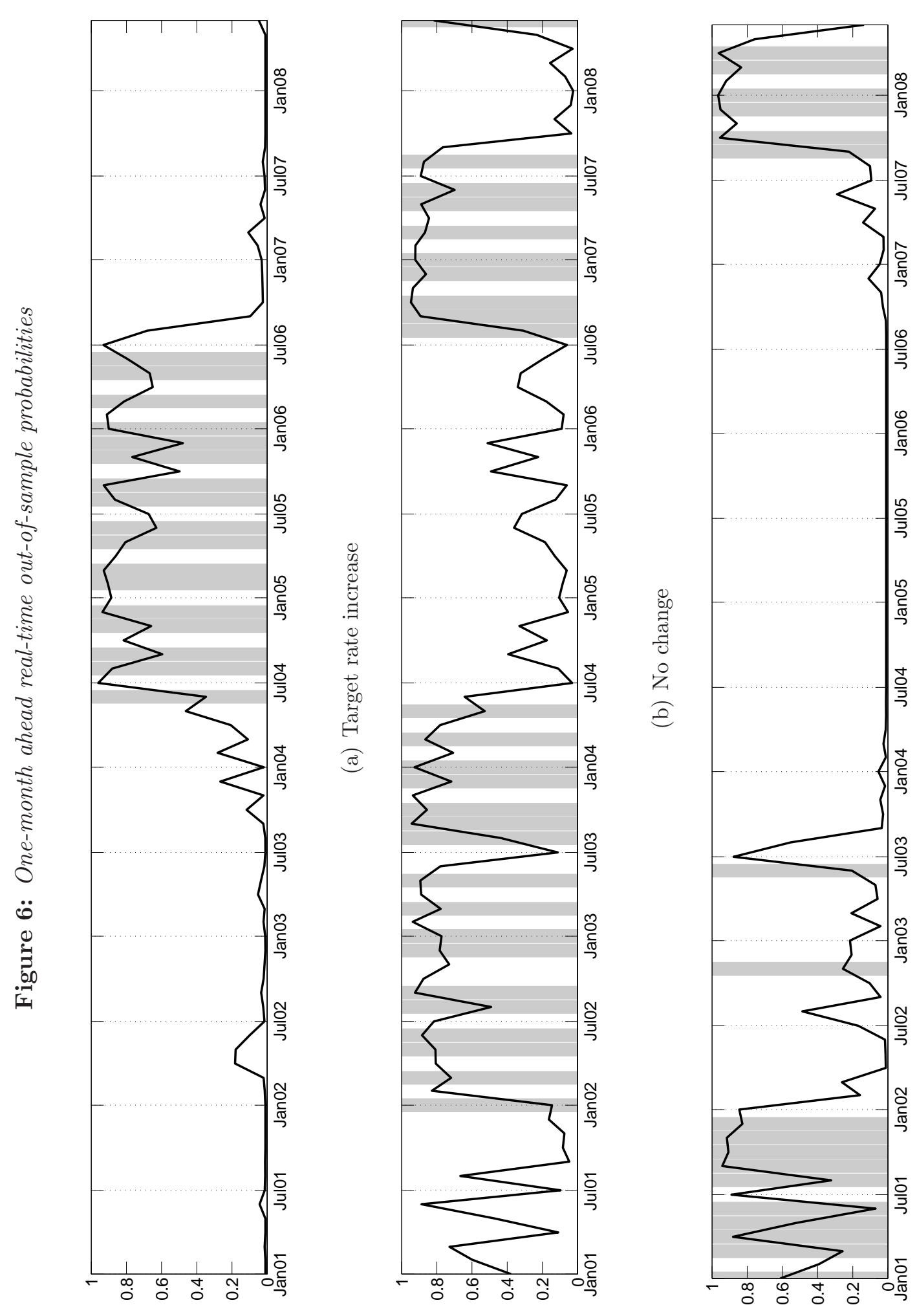

II

ت

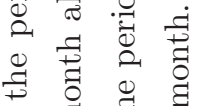

苛

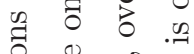

券的 ह

ช

苞

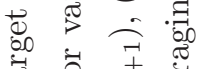

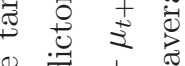

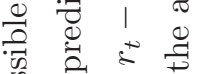

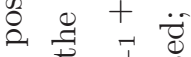

$8+\frac{0}{8}$

ఫृ

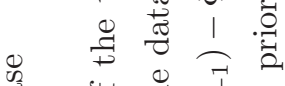

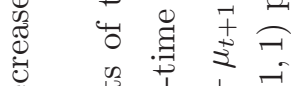

ర)

$\stackrel{0}{0}=0$

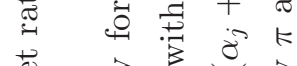

焉

(-)

范

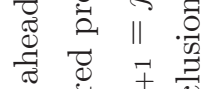

هि

जि

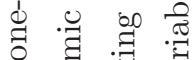

음

형 웧

敢 击

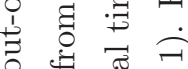

- 0

苛.

प气

ज

象 0

胥

$\ddot{\theta} \cong$.

焉莺

之. 
Figure C.1: Alternative $y_{t}:$ Federal funds target rate, latent target rate and thresholds

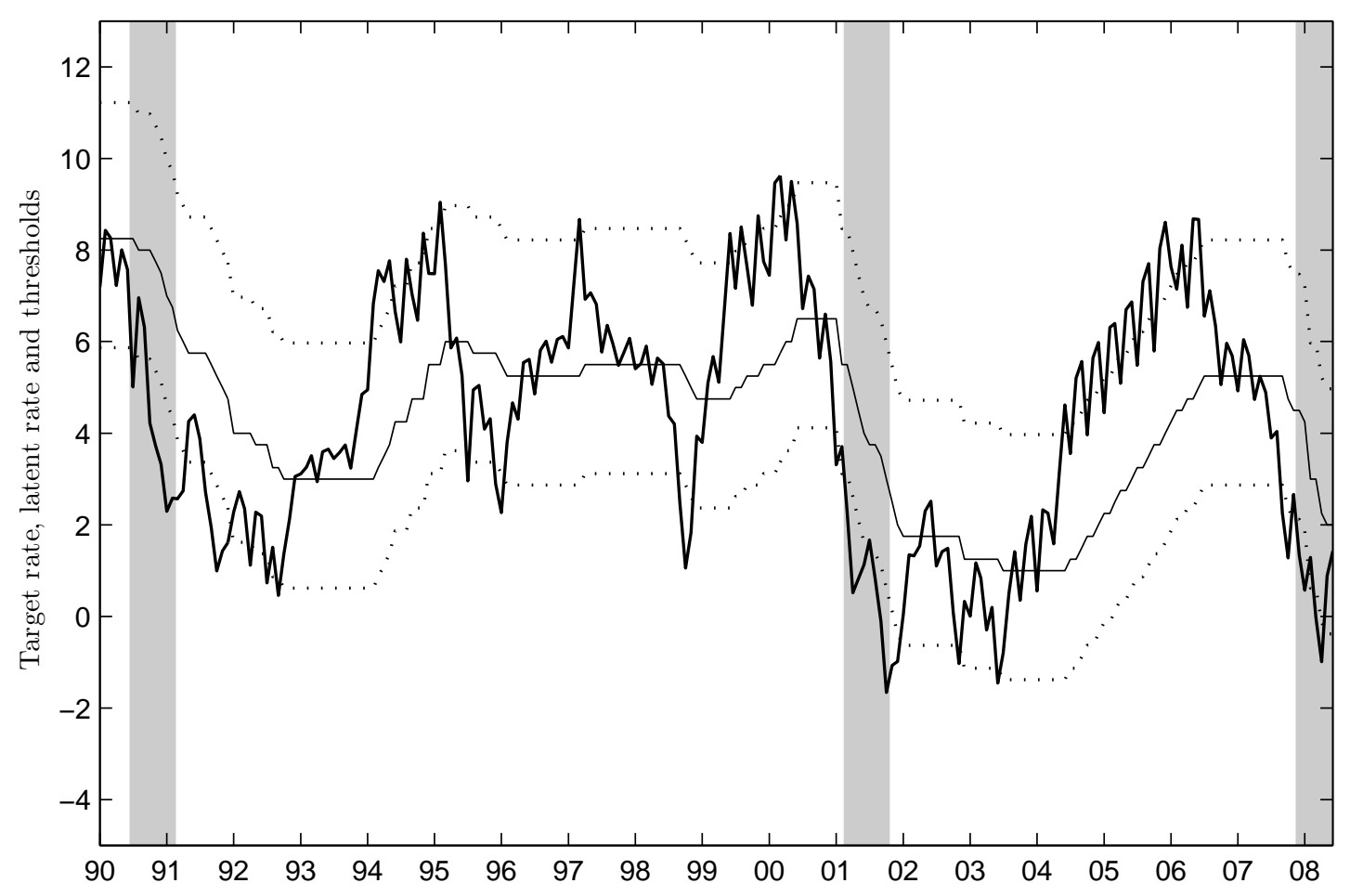




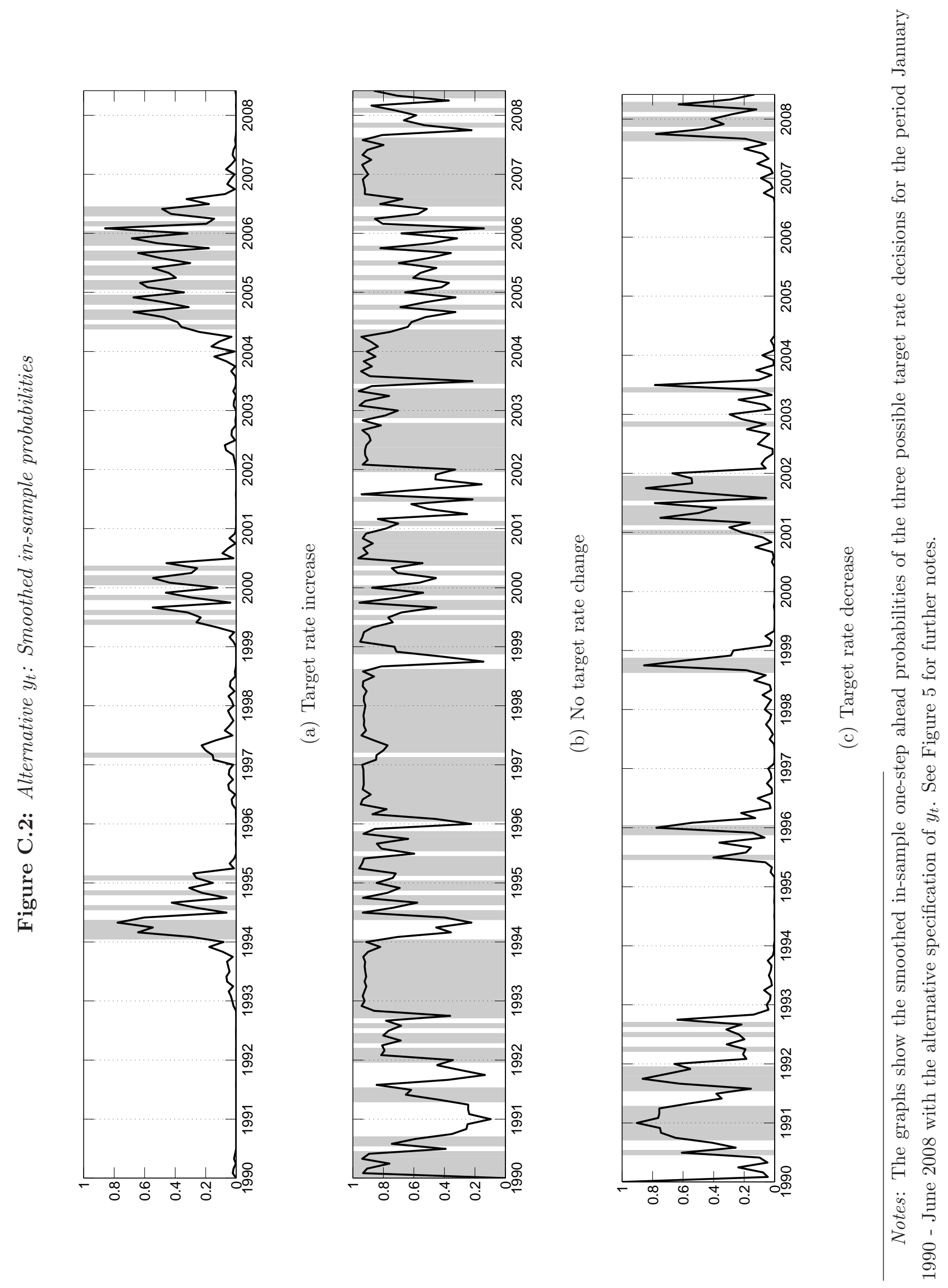



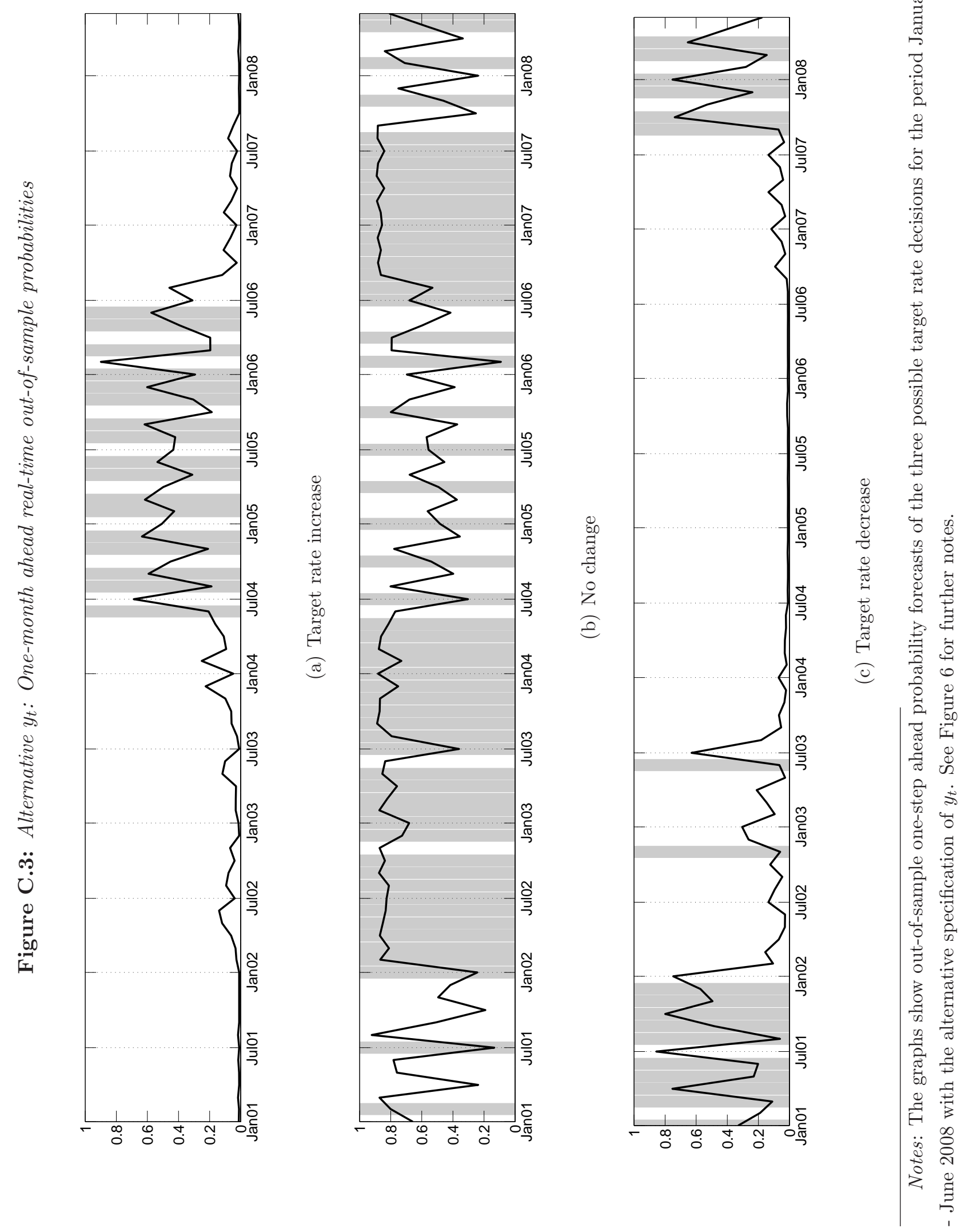